\title{
Les « ichtyolites » (Actinopterygii) de la collection Jean-Baptiste Beurard (1745-1835) : intérêt historique et redécouverte de la série type d'Armigatus brevissimus (Blainville, 1818) du Cénomanien du Liban
}

\author{
Arnaud Brignon* \\ 5 villa Jeanne d'Arc, 92340 Bourg-la-Reine, France
}

Reçu le 8 février 2021 / Accepté le 23 juin 2021 / Publishing online: 15 July 2021

\begin{abstract}
Résumé - Cet article souligne le rôle Jean-Baptiste Beurard dans l'histoire de la paléoichtyologie. Ancien chanoine de la cathédrale de Toul, il trouva après les affres des débuts de la Révolution un emploi d'agent du gouvernement attaché à l'administration des mines entre 1794 et 1815 . Chargé de la surveillance des mines de mercure dans les nouveaux départements annexés par la France sur la rive gauche du Rhin, il redécouvrit en juillet 1799 le fameux gisement d'«ichtyolites» (poissons fossiles) imprégnés de cinabre de Münsterappel daté du Permien inférieur. Il envoya plusieurs spécimens dans des collections institutionnelles parisiennes. Des représentants de l'espèce Paramblypterus duvernoy (Agassiz, 1833) (Actinopterygii, Amblypteridae), probablement envoyés par Beurard avant 1809 d'après le témoignage de Barthélémy Faujas de Saint-Fond, ont été identifiés dans les collections du Muséum national d'Histoire Naturelle, Paris. Un autre spécimen ayant appartenu de manière incontestable à Beurard est conservé dans les collections Géosciences de Sorbonne Université et revêt un intérêt historique tout particulier. Beurard possédait également dans sa collection deux échantillons de poissons fossiles du Cénomanien de Haqel dans l'actuel Liban, qu'il avait reçu de son neveu Claude Charles Harmand (1784-1847), officier de marine, en 1817. À partir d'eux, Henri Marie Ducrotay de Blainville décrivit deux espèces qu'il nomma Clupea beurardi Blainville, 1818a et Clupea brevissima Blainville, 1818a. La première n'est plus considérée comme valide et la seconde est aujourd'hui assignée au genre Armigatus Grande, 1982 (Actinopterygii, Clupeomorpha) dont elle est l'espèce type. Un de ces spécimens est conservé au Natural History Museum, Londres, et provient de la collection de William Willoughby Cole (1807-1886), comte d'Enniskillen, qui avait acquis une partie de la collection Beurard. Étiqueté par erreur Clupea beurardi par Beurard, cet échantillon porte en réalité les quatre syntypes d'Armigatus brevissimus. Un lectotype est désigné ici pour fixer le statut de cette espèce, certainement la plus abondante et la plus iconique du gisement de Haqel.
\end{abstract}

Mots clés : Histoire de la paléontologie / Actinopterygii / Permien / Cénomanien / Allemagne / Liban

\begin{abstract}
The "ichtyolites" (Actinopterygii) from the collection of Jean-Baptiste Beurard (17451835): historical significance and rediscovery of the type series of Armigatus brevissimus (Blainville, 1818) from the Cenomanian of Lebanon. This article highlights the role of Jean-Baptiste Beurard in the history of palaeoichthyology. A former canon of the Cathedral of Toul, he became a government employee attached to the mine administration between 1794 and 1815 after the turmoil of the early revolutionary period. He was in charge of supervising the mercury mines in the new departments annexed by France on the left bank of the Rhine. In July 1799, he rediscovered in the area of Münsterappel the famous Lower Permian deposit of "ichtyolites" (fossil fish) impregnated with cinnabar. He sent several specimens to institutional collections in Paris. Representatives of the species Paramblypterus duvernoy (Agassiz, 1833) (Actinopterygii, Amblypteridae), probably sent by Beurard before 1809 according to the testimony of Barthélémy Faujas de Saint-Fond, have been identified in the collections of the Muséum national d'Histoire Naturelle, Paris. Another specimen that indisputably belonged to Beurard is preserved in the Geosciences Collections of the Sorbonne University (Paris) and is of particular historical interest. Beurard also possessed
\end{abstract}

*Auteur correspondant : arnaud.brignon@yahoo.com 
in his collection two samples of fossil fish from the Cenomanian of Haqel, in present-day Lebanon, received from his nephew Claude Charles Harmand (1784-1847), naval officer, in 1817. Based on those specimens, Henri Marie Ducrotay de Blainville described and named the two species Clupea beurardi Blainville, 1818a, and Clupea brevissima Blainville, 1818a. The first one is no longer considered valid and the second one is now assigned to the genus Armigatus Grande, 1982 (Actinopterygii, Clupeomorpha), of which it is the type species. One of these specimens is kept at the Natural History Museum, London, and comes from the collection of William Willoughby Cole (1807-1886), Earl of Enniskillen, who had acquired part of the Beurard collection. Erroneously labelled Clupea beurardi by Beurard, this sample actually bears the four syntypes of Armigatus brevissimus. A lectotype is designated here to settle the identity of the species, which is certainly the most abundant and iconic one found in the Haqel deposit.

Keywords: History of palaeontology / Actinopterygii / Permian / Cenomanian / Germany / Lebanon

\section{Introduction}

L'histoire des débuts de la paléoichtyologie, ou la science des «poissons» fossiles, est étroitement liée aux collectionneurs privés qui permirent aux scientifiques de leur temps de disposer d'un matériel abondant et facilement consultable (Davies, 1970; Gaudant et Bouillet, 1997; Brignon, 2014, 2017b, 2019a ; Capasso, 2014 ; Bernard et Smith, 2015 ; Smith, 2015). L'étude historique des anciennes collections paléontologiques et l'exploitation de toutes les sources bibliographiques et manuscrites disponibles permettent souvent de résoudre des questions nomenclaturales délicates, voire de retrouver du matériel type que l'on croyait perdu depuis longtemps (Brignon, 2015, 2016a, 2016b, 2019b, 2019c ; Young et al., 2020). Dans cette perspective, cet article s'intéresse à un français d'origine lorraine, Jean-Baptiste Beurard (1803) qui fut le premier à publier, en 1803, un article entièrement consacré à des poissons (Actinopterygii) découverts dans les mines de mercure du Palatinat qui venait d'être annexée à la France suite aux victoires de l'armée révolutionnaire. Beurard possédait également des téléostéens du Cénomanien de Haqel au Liban qui servirent de types aux deux premières espèces décrites provenant ce célèbre gisement (Blainville, 1818a, 1818b). L'histoire de ces découvertes est détaillée, des spécimens trouvés il y a plus de deux siècles sont présentés et leur importance scientifique est analysée.

\section{Biographie}

Quatrième d'une grande fratrie de treize enfants, Jean-Baptiste Beurard naquit à Nancy le 5 novembre 1745 (Quérard, 1827 : $320,321)^{1}$. Il est le fils d'Agnès Angélique Martin ${ }^{2}$ et de Jean Claude Beurard ${ }^{3}$, écuyer, conseiller du roi, greffier en chef civil et criminel au Parlement de Lorraine, secrétaire perpétuel de la Chambre des consultations. Destiné à une carrière religieuse, Jean-Baptiste Beurard fut reçu chanoine de la cathédrale de Toul le 27 juillet 1761 (Benoît, 1884: 66).

\footnotetext{
1 AD54, registres paroissiaux, paroisse Saint-Sébastien, baptêmes, 1730-1750, 5 Mi 394/R 68.

2 Née le 13 octobre 1718 à Commercy (Meuse), paroisse SaintPantaléon, et décédée le 9 mars 1785 à Nancy, paroisse Saint-Nicolas. Elle épousa Jean Claude Beurard le 3 novembre 1739 à Vicherey.

3 Né le 5 octobre 1712 à Vicherey dans les Vosges et décédé le $1^{\mathrm{er}}$ juillet 1786 à Nancy, paroisse Saint-Nicolas.
}

Ses études lui permirent d'obtenir une licence en droit ${ }^{4}$. D'après l'abbé Laurent Chatrian (1732-1814), curé de SaintClément (Guillaume, 1867 : 316-319), Beurard faisait partie de ces «jeunes chanoines, nommés par la faveur royale» qui «s'en viennent, à Toul, scandaliser les anciens et afficher leurs manières mondaines en se promenant publiquement avec des dames». Critique envers son cadet, Chatrian poursuivait dans son journal: "M. Beurard va même au bal pendant le carnaval» (cité par Mathieu, 1879: 330)!

En voyage en Italie, il fut reçu membre de l'Académie des Arcades de Rome le 4 mars 1779 (Tutot, 1779: 292). Beurard œuvrait pour rentrer en possession du prieuré de SaintThiébault sous Bourmont ${ }^{5}$, dans le diocèse de Toul, vacant depuis le décès de son dernier titulaire ${ }^{6}$. Il avait obtenu dans cette démarche l'accord de l'évêque de Saint-Dié, suivi d'une décision favorable du pape Pie VI le 2 août 1779. La congrégation de Saint-Vanne et de Saint-Hydulphe, ordre de Saint-Benoît, dont dépendait le prieuré en question, s'opposa cependant à la nomination de Beurard. Il s'ensuivit un long procès pour le débouter. Ses détracteurs reprochaient à Beurard de s'être «rendu indigne» et que cette indignité résultait «de faits graves relativement aux mœurs ${ }^{7}$. Néanmoins, ces faits n'étaient pas judiciairement prouvés ${ }^{8}$ et Beurard pouvait compter sur le soutien de l'évêque de Toul qui ne s'était «point opposé à ce qu'il jouït paisiblement jusqu'à présent de sa présence canoniale de Toul» et qui lui avait donné «un visa dans lequel il reconnoit et atteste ses bonnes vie, moeurs et capacités ». Quoi qu'il en soit, un vice de procédure dans l'attribution du prieuré à Beurard fut reconnu et le prieuré de Saint-Thiébault fut finalement confié à Joseph Febvet, visiteur de la congrégation.

La Révolution de 1789 lui fit perdre sa position ecclésiastique et renoncer à des «revenus considérables》 comme il l'écrivait lui-même en juillet 1815 au comte Louis-Mathieu Molé (1781-1855), directeur général des ponts et chaussées et des mines 9 . Fuyant la persécution, il dut s'exiler en 1790. Après la chute de Robespierre et la fin de la Terreur, Beurard retourna en France à la fin de l'année 1794. Ses

\footnotetext{
4 AD55, 4H100/198-199 (Becquart et Colnat, 1958: 130).

5 Actuelle département de la Haute-Marne.

6 AD55, 4H100/199, 202.

7 AD55, 4H100/202.

8 AD55, 4H100/208.

9 AN, Travaux publics. Personnel de l'Administration centrale: dossiers individuels. Lettre de Beurard au comte Louis-Mathieu Molé, Paris, 25 juillet 1815, F/14/11401.
} 
connaissances en minéralogie lui permirent d'obtenir le 21 frimaire an 3 (11 décembre 1794) un poste temporaire à l'Agence des mines de la République en tant qu'adjoint de Johann Gottfried Schreiber (1746-1827), inspecteur des mines. Dès le mois de février 1795 , le Comité de salut public mit à sa disposition une voiture pour rejoindre les représentants du peuple de l'armée du Rhin afin de visiter les mines du Palatinat (Aulard, 1910 : 169). Sa mission consistait à évaluer la richesse en minerai des territoires conquis par l'armée française et de mettre en place l'exploitation des mines jugées rentables. Par ordre d'une commission du Ministère de l'Intérieur, Beurard obtint le 18 brumaire an 4 (9 novembre 1795) un poste fixe d'agent du Gouvernement «pour résider seul sur les mines de mercure des Palatinat et Duché de Deux Ponts afin d'en surveiller immédiatement les exploitations $\gg{ }^{9}$, comme l'indique ses états de service (Fig. 1). Outre ce travail de régisseur, il assurait les rôles de trésorier et de fondé de pouvoir des actionnaires des mines de mercure (Sainte-Claire Deville, 1929: 20). Sa tâche n'était pas simple comme il en témoignait à Louis-Mathieu Molé: "dès les premières années de mon service, des mouvemens [sic] d'armée m'ont placé dans le danger imminent de perdre la vie et fait dépouiller trois fois de tout ce que je possédais. [...]. Les anciens chefs de cette administration savent que pour parvenir à maintenir en activité les exploitations de mines de mercure, au milieu de tant de difficultés et entraves sans cesse renaissantes, il m'a fallu braver bien des dangers et me condamner à toutes sortes de privations $\rangle^{10}$.

Par une décision du Ministère de l'Intérieur datée du 24 prairial an 11 (13 juin 1803), Beurard fut envoyé à Hanovre puis à Clausthal avec le titre de commissaire du Gouvernement adjoint de l'ingénieur des mines Antoine-Marie Héron de Villefosse (1774-1852) (Héron de Villefosse, 1804 ; Dettmer, 2015). Alors même que l'armée française occupait cette région, ils avaient pour mission de « veiller à la conservation des mines et usines du Harz et faire réserver au profit de la France les droits pécuniaires revenans [sic] au roi d'Angleterre ${ }^{9}$. Le 26 prairial an 11 (15 juin 1803), Francois Pierre Nicolas Gillet de Laumont (1747-1834), inspecteur des mines, écrivait au jeune ingénieur Héron de Villefosse pour lui expliquer les raisons pour lesquelles Beurard avaient été choisi et pour lui donner quelques conseils sur la manière de se comporter avec son nouveau collaborateur de 29 ans son aîné ${ }^{11}$ : «Le Cn [Citoyen] Beurard paroit un peu effraié ${ }^{12}$. J'ai cherché à le rassurer, son expérience, la mission qu'il a déjà eu comme commissaire du gouvernement avec le Cn Schreiber dans le Palatinat lorsque les armées y étoient encore, sont des titres d'après les quels nous pensons qu'il remplira d'une manière distinguée cette nouvelle marque de confiance du gouvernement. Beurard s'exprime beaucoup mieux par écrit que verbalement, il entend bien

10 AN, Travaux publics. Personnel de l'Administration centrale : dossiers individuels. Lettre de Beurard au comte Louis-Mathieu Molé, Paris, 30 juillet 1815, F/14/11401.

11 Archives de Gillet de Laumont, Bibliothèque Mines ParisTech, brouillon d'une lettre de Gillet de Laumont à Heron de Villefosse à propos d'une mission que celui-ci va effectuer en Allemagne, dans la région de Harz, avec Beuvard [sic], 26 prairial an 11.

12 Les passages biffés ont été supprimés dans cette transcription. l'allemand, il a la melieure [sic] volonté de l'activité. Je l'ai assuré que quoiqu'il ne soit désigné que comme commissaire adjoint vous auriez pour lui les égards dus à son age [sic], à son expérience. Dans le fait mon ami vous estes [sic] bien jeune pour avoir le pas sur un viellard [sic] (j'appelle ainsi ceux de mon age), mais vous devez penser que vous le devez à votre qualité d'ingénieur des mines. Je vous engage donc à ménager son amour propre si elle étoit dans le cas de se trouver compromise. Il est de même que vous commissaire du gouvernement, mais vous estes réunis pour faire le plus de bien possible, pour résister à tout ce qui pouroit altérer l'exploitation de ces mines, ou tourmenter les braves et laborieux mineurs. Je ne suis pas inquiet de l'honneteté [sic] et de la fermeté avec la quelle vous vous présenterez vis avis [sic] des généraux et je ne doute pas que vous ne trouviez dans Beurard un collègue qui vous secondera efficacement auprès de ces bons allemands, il traduira tout, il parlera aux mineurs en homme habitué avec eux et la jeunesse tempérée par la viellesse [sic] fera des actes qui tiendront de la vigueur de l'âge mur. Voilà mon ami ce que j'augure de votre importante mission. N'oubliez pas de former une collection minéralogique pour le conseil.»

Le 22 nivôse an 13 (12 janvier 1805), Jean-Baptiste Nompère de Champagny (1756-1834), ministre de l'Intérieur, rappela Beurard à ses premières fonctions «d'agent $d u$ gouvernement sur les mines de mercure» dans le Palatinat. En 1807 , le poste de bibliothécaire-interprète de l'administration des mines étant vacant, le même ministre suggéra au conseil des mines de le confier à Beurard «pour ne pas laisser échapper l'occasion de retenir attaché à l'établissement un homme qui lui a été utile, qui peut lui être beaucoup plus utile encore pour les traductions, et qui mérite récompense ${ }^{13}$. Le conseil jugea cependant nécessaire de maintenir Beurard à la surveillance des mines de mercure. Beurard avait néanmoins obtenu du ministre une lettre officielle datée du 17 juillet 1807 lui garantissant qu'au cas où sa place d'agent du Gouvernement viendrait à être supprimée, celle de bibliothécaireinterprète lui serait assurée. Beurard resta jusqu'au mois d'août 1812 dans le Palatinat où il était posté à Meisenheim (Anonyme, 1809 : 302). Il fut ensuite chargé par le ministre des Finances de se «rendre sur les Salines anséatiques [sic] et coopérer, en qualité de chef des liquidations aux opérations du Commissaire spécial (M. Haudry de Soucy) ${ }^{14}$ chargé de leur organisation et de leur régie »"

Avec la chute du Premier Empire, Beurard rentra en France. Le $1^{\text {er }}$ août 1814 , comme l'avait promis Nompère de Champagny dans sa lettre datée du 17 juillet 1807 , il fut nommé bibliothécaire et traducteur à la direction générale des mines par le ministre de l'Intérieur de l'époque, l'abbé François-Xavier-Marc-Antoine de Montesquiou-Fezensac (1756-1832). Cependant une partie non négligeable de son salaire lui était soustrait pour payer la retraite de l'ancien bibliothécaire Eugène Louis Melchior Patrin (1742-1815) auquel il succédait. N'ayant que ce modeste traitement pour vivre, Beurard prit la plume pour plaider sa cause auprès du

\footnotetext{
13 AN, écrit de Jean-Baptiste Nompère de Champagny cité par Beurard. Lettre de Beurard au comte Louis-Mathieu Molé, Paris, 25 juillet $1815, \mathrm{~F} / 14 / 11401$.

14 André Haudry de Soucy (1765-1844), base Léonore, dossier LH/ $1271 / 40$
} 


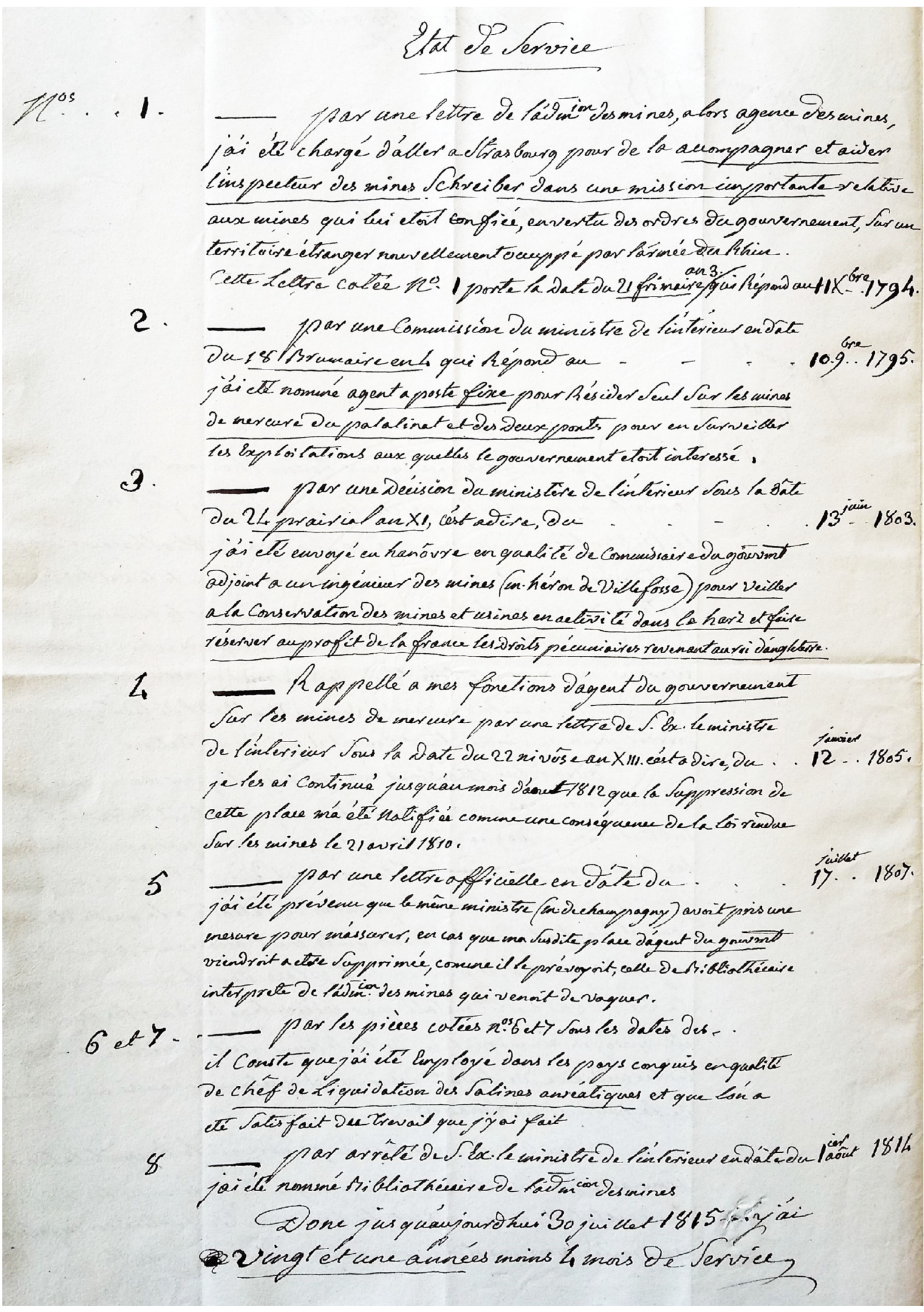

Fig. 1. États de service de Jean-Baptiste Beurard en tant qu'agent du Gouvernement français entre 1794 et 1815 (AN F-14-11401); manuscrit écrit par l'intéressé et envoyé au directeur général des ponts et chaussées et des mines, le comte Louis-Mathieu Molé (1781-1855).

Fig. 1. Service record of Jean-Baptiste Beurard as an agent of the French government between 1794 and 1815; handwritten manuscript by Beurard sent to the chief director of the civil engineering and mine administration, Louis-Mathieu Molé (1781-1855). 
comte Louis-Mathieu Molé, directeur général des ponts et chaussées et des mines. Il s'exprimait en ces termes : «[...] je suis attaché à l'administration des mines depuis vingt et une années. Ses cartons, de même que ceux des ministères de l'intérieur et des finances renferment les preuves que pendant cette période, j'ai rendu des services utiles qui m'ont exposé souvent à de grands dangers et $m$ 'ont fait éprouver des pertes considérables, légalement constatées, pour lesquelles même déjà depuis bien longtemps, il m'avoit été promis des indemnités que je n'ai jamais reçues. Si vous daignés [sic] interroger les anciens et principaux chefs du corps des mines, j'ai lieu de croire qu'ils vous répéteront ce qu'ils m'ont souvent dit et écrit, particulièrement lorsque j'ai quitté le palatinat, que jamais ils n'avoient varié d'opinion sur mon compte. Victime de toutes les tempêtes politiques depuis 25 ans; sans autre resource [sic] aujourd'hui que mon modique traitement partagé par un viellard infirme ${ }^{15}$; agé moi-même de 70 ans moins 3 mois, mais encore plein d'ardeur pour le travail, je laisse à votre justice à prononcer sur mon sort, auquel est lié celui d'un autre malheureux ${ }^{15}$, et je $m^{\prime} y$ confie ${ }^{9}$. En écrivant ces mots, Beurard ne se doutait pas qu'au même moment le comte Louis-Mathieu Molé venait de prendre un arrêté ordonnant la réunion de la bibliothèque de l'administration des mines avec celle des ponts-et-chaussées et nommant à leur tête l'abbé Nicolas Halma (1755-1828), l'ancien bibliothécaire de l'impératrice Joséphine $^{16}$. Se retrouvant maintenant sans emploi, il n'avait pour vivre qu'une rente de 300 francs obtenue «du sacrifice $q u$ '[il avait] dî faire» au début de l'année 1815 de «[ses] collections minéralogiques qui faisaient tout [son] bonheur $\gg{ }^{10}$. Il n'avait maintenant d'autre choix que de demander à Molé de lui octroyer une pension de retraite pour ses vingtet-un ans de bons et loyaux services. Louis XVIII étant à nouveau au pouvoir, Beurard rappelait opportunément : «j'ai constamment refusé d'accepter et de signer l'acceptation de l'acte additionnel qui excluait du trône une Dynastie dont les malheurs seuls ont fait les miens $\gg{ }^{10}$. Une pension lui fut accordée par une ordonnance du roi datée du 8 août 1815 . «Ayant appartenu à l'état ecclésiastique de l'ancienne France », une pension complémentaire lui fut versée par une seconde ordonnance datée du 26 février $1823^{17}$.

Beurard demeurait au $n^{\circ} 31$ rue Servandoni dans l'ancien $\mathrm{XI}^{\mathrm{e}}$ arrondissement ${ }^{18}$ de Paris puis au $\mathrm{n}^{\mathrm{o}} 5$ quai de la Tournelle dans l'ancien XII ${ }^{\mathrm{e}}$ arrondissement ${ }^{19}$ (Savardan, 1836 : 266). Il décéda à son domicile le 9 août 1835, dans sa quatre-vingtdixième année $\mathrm{e}^{20}$. Un témoignage relate que «sa santé était encore parfaite» en 1833 (Savardan, 1836: 266).

\footnotetext{
15 Eugène Louis Melchior Patrin (1742-1815).

16 Le reçu officialisant la remise des clefs de la bibliothèque de l'administration des mines à Halma par Beurard est conservé dans les Archives de Gillet de Laumont (dossier 2), Bibliothèque Mines ParisTech.

17 Bulletin des Lois, 1823, série 7, 16(593bis) : 46-49.

18 Cette rue est aujourd'hui située dans le 6 e arrondissement de Paris.

19 Aujourd'hui située dans le $5^{\mathrm{e}}$ arrondissement.

20 AD75, V3E/D 112.
}

\section{Les travaux de Beurard et son implication dans les sociétés savantes}

$\mathrm{Au}$ cours de sa première mission dans le Palatinat entre février 1795 et juin 1803, Beurard rédigea plusieurs rapports qui furent publiés dans le Journal des Mines. D'autres rapports inédits, restés sous forme manuscrite, sont également conservés aux Archives nationales (Engerand, 1920) ${ }^{21}$. Ces travaux permettent de suivre ses investigations sur les gisements de cuivre, de charbon, de plomb et de mercure dans les départements français du Mont-Tonnerre et de la Sarre créés en 1797 (Fig. 2). Il visita et étudia notamment la mine de cuivre de Fischbach, dans l'actuel arrondissement de Birkenfeld (land de Rhénanie-Palatinat). Il estimait d'après ses calculs que la mine était rentable et recommandait donc au Gouvernement son exploitation (Beurard, 1797). Sa mission principale étant de veiller à la bonne productivité des mines de mercure, il avait établi un inventaire détaillé des différents filons et, pour chacun d'eux, avait consigné sa situation géographique et stratigraphique, son mode d'extraction et son rendement (Beurard, 1798a). Il rédigea également un rapport sur les mines de houille des environs de Meisenheim (Beurard, 1798b).

Beurard est l'auteur d'une notice sur des poissons fossiles trouvés dans le Permien inférieur de Münsterappel dont il sera question plus loin (Beurard, 1803 ; Brignon, 2014). Fort de sa maîtrise de l'allemand, il publia en 1809 un dictionnaire allemand-français des termes propres à l'exploitation des mines, la métallurgie et la minéralogie qui fut réédité en 1819 (Beurard, 1809, 1819a). Il traduisit également un article en allemand de Johann Jakob Nöggerath (1788-1877) sur la «Description minéralogique du gisement de la Braunkohle (houille brune), dans la colline de Putzberg, près de Friesdorf, département de Rhin-et-Moselle » (Nöggerath, 1811). À son retour en France en 1814 et la prise de sa fonction de bibliothécaire à la direction générale des mines, Beurard publia à nouveau une série d'articles dans le Journal des Mines, à savoir, une notice sur les houillères de Borgloh, dans l'arrondissement d'Osnabrück, extraite d'un rapport qu'il avait rédigé en 1812 (Beurard, 1814a), des notes sur les salines de Lunebourg et de Bad Rothenfelde (Beurard, 1814b, 1814c), ainsi que l'extrait d'un rapport sur les méthodes d'extraction du mercure (Beurard, 1815).

Après sa mort, il laissait chez lui plusieurs manuscrits inédits qui figurent dans l'inventaire de sa succession faite en septembre $1835^{22}$. Il avait notamment rédigé un «Précis sur le Hartz $»^{23}$, un «Manuel minéralogique» par ordre alphabétique, un volumineux «Catalogue raisonné de minéraux qui donne spécialement la description oryctographique de toutes

\footnotetext{
21 AN, principaux documents des Archives nationales relatifs aux régions de la Sarre et de la rive gauche du Rhin, F/14/1076/1.

22 AN, minutes et répertoires du notaire Antoine Pierre Le Fer, 12 septembre 1834-16 février 1858 (étude XCVIII), MC/ET/XCVIII/ 969.

${ }^{23}$ Ce travail rédigé à Clausthal en janvier 1805, juste avant son retour dans le Palatinat, est mentionné dans les bibliographies de Beurard sous le titre « Mémoire historique et descriptif sur le Hartz » (Quérard, 1827; Durozoir, 1843).
} 

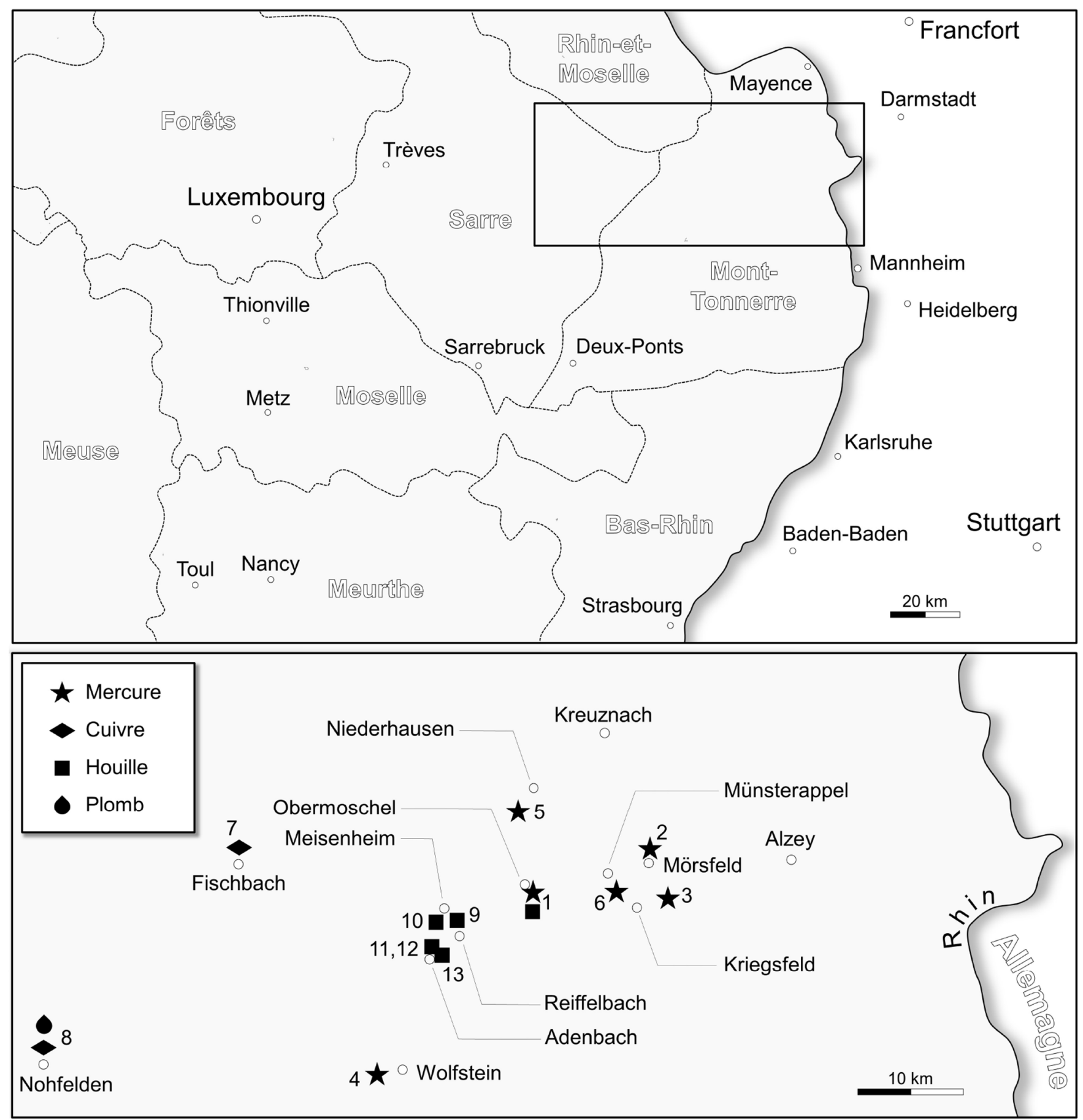

Fig. 2. En haut, carte des départements français créés en 1797 sur la rive gauche du Rhin. En bas, agrandissement de l'encart sur lequel sont indiquées les mines visitées et étudiées par Beurard dans les départements du Mont-Tonnerre et de la Sarre (Beurard 1797, 1798a, 1798b, 1803; AN, F/14/1076/1). 1: mines de Moschellandsberg; $2:$ mines de Mörsfeld; $3:$ mines du Spitzenberg; $4:$ mines de Wolfstein; $5:$ mines du «Limberg» [Lemberg]; 6: mines de Münsterappel; 7: mine de Fischbach; 8: mine de Nohfelden; 9: mine de Reiffelbach; 10: mine de Blochersberg; 11 : mine de Ludwig; 12 : mine de Saint-Jacques ; $13:$ mine de Hahlcreuzer.

Fig. 2. Top, map of the French departments created in 1797 on the left bank of the Rhine. Bottom, enlarged view of the insert showing the mines visited and studied by Beurard in the Mont-Tonnerre and Sarre departments (Beurard 1797, 1798a, 1798b, 1803; AN, F/14/1076/1). 1: mines of Moschellandsberg; 2: mines of Mörsfeld; 3: mines of Spitzenberg; 4: mines of Wolfstein; 5: mines of "Limberg" [Lemberg]; 6: mines of Münsterappel; 7: mine of Fischbach; 8: mine of Nohfelden; 9: mine of Reiffelbach; 10: mine of Blochersberg; 11: mine of Ludwig; 12: mine of Saint-Jacques; 13: mine of Hahlcreuzer. 
les variétés de minerai de mercure que fournissent les mines $d u$ Palatinat et du duché de Deux-Ponts, ainsi que des autres minéraux qui les accompagnent; avec un essai sur la géologie des contrées où elles se trouvent » (Quérard, 1827), plusieurs traductions d'ouvrages allemands comme celle de la «Description minéralogique de la chaine du Vogelsgebirg dans le landgraviat de Hesse-Darmstadt » de Klipstein (1790) et celle de la «Description minéralogique des contrées les plus remarquables par leurs mines, dans le duché de Deux-Ponts et dans le Palatinat \& $\&$ » de Ferber (1776). Il avait également rédigé plusieurs journaux de ses voyages en Italie, en Allemagne, en Hollande, en Suisse, dans les Alpes et les montagnes des Vosges (Quérard, 1827).

Le 18 novembre 1802, il avait été reçu membre associé correspondant de l'Académie des Sciences, Arts et BellesLettres de Dijon (Antoine et Toussaint, 1836: 173) à laquelle il communiqua un mémoire «sur la manière de faire les recherches des mines dans le Palatinat et d'en commencer les exploitations et sur les diverses méthodes employées pour l'extraction du mercure de sa gangue » (Anonyme, 1805 : 22). Outre son admission à l'Académie des Arcades de Rome en 1779, il fut reçu membre de la Société royale des sciences de Göttingen, de la Société minéralogique de Jena, de la Société des recherches utiles de Trèves, de l'Académie de la Vettéravie (Wetterau) (Quérard, 1827; Durozoir, 1843) et de la Société royale d'Arras pour l'encouragement des Sciences, des Lettres et des Arts à laquelle il avait soumis une note sur la ville de Hambourg (Beurard, 1819b).

\section{Le «cabinet de minéralogie » de Beurard}

Beurard consacrait ses loisirs à enrichir son cabinet de minéralogie et à travailler les pierres, occupation qu'il appelait son «chasse-diable» et l'aidait apparemment à se détourner des «mauvaises pensées» comme l'écrivait le médecin Auguste Savardan (1792-1867) à l'évêque du Mans, Monseigneur Bouvier: "l'agriculture, la physique, la chimie, l'histoire naturelle, l'archéologie, la peinture, la musique comptent, dans le clergé de nos campagnes, quelques adeptes et ces prêtres-là ne sont pas les moins bons. Il en est qui ont pris à tâche de doter leur presbytère d'une collection de tous les produits naturels de la paroisse et on ne saurait nier l'importance que pourrait avoir l'établissement de pareils musées dans toutes les communes. L'étude des sciences et des arts vient donc très-bien en aide au bréviaire pour conjurer le malin esprit et résister à l'entraînement de ses tentations. C'était encore, à plus de 80 ans, l'avis d'un ancien chanoine de Toul, l'abbé Beurard, auteur d'un bon dictionnaire de minéralogie français-allemand: possesseur d'un riche cabinet de minéralogie, il passait une grande partie de son temps à polir de belles pierres et à en composer avec beaucoup d'art, de petits meubles ou des tableaux. Un jour que nous lui témoignions notre surprise de son assiduité à ce travail, quelquefois assez pénible: qu'aurais-je donc pour me défendre contre les mauvaises pensées, nous répondit-il avec une spirituelle gaité? Tenez, mon ami, ajouta-t-il, il y a tantôt vingt ans que ce travail-là est mon meilleur chasse-diable» (Savardan, 1846 : 42 ; 1866 : 453). La collection minéralogique de Beurard était réputée en son temps. Le géologue Jean-Baptiste d'Omalius-d'Halloy (1808: 450) la qualifiait de «magnifique». Beurard connaissait le collectionneur de fossiles et de minéraux Étienne de Drée (1760-1848) avec qui il échangeait des échantillons (Léman, 1815: 66). Il envoyait régulièrement des minéraux et des fossiles pour les collections du Muséum d'Histoire naturelle à Paris ainsi que pour celles de l'école des Mines (Faujas de Saint-Fond, 1809 : 349; Deleuze, 1823: 344). Dans la seconde partie de son «Tableau méthodique des espèces minérales » publiée en 1813, JeanAndré-Henri Lucas (1780-1825), garde des galeries du Muséum, mentionne ainsi des échantillons de «mercure argental » du département de Deux-Ponts que Beurard avait offerts au Muséum. Il est à signaler que les collections paléontologiques du MNHN possèdent une portion de maxillaire de crocodilien marin du Jurassique des Vaches Noires, polie sur une face, sur laquelle est écrit à l'encre « $d u$ cabinet de Mr Beurard» (Brignon, 2017a, 2020). Notons enfin que le catalogue manuscrit des ossements fossiles de vertébrés placés dans les galeries de géologie et de minéralogie (p. 1317, numéro ancien AC 8591) indique que le MNHN possédait en 1861 un os long de plésiosaure de provenance inconnue qui avait également appartenu à Beurard.

En 1828, Beurard avait fait de Claude Frédéric Donop $(1796-1865)^{24}$, son légataire universel. Alors capitaine au corps royal d'état-major, Donop était sorti de l'école Polytechnique en 1813. Son père, Frédéric Guillaume Donop (1773-1815), général de l'Empire, avait été tué à la bataille de Waterloo. Sous le Second Empire, Claude Frédéric Donop fut reçu commandeur de l'ordre impérial de la Légion d'Honneur en 1854. Il termina sa carrière comme intendant militaire à la division d'Alger. L'inventaire de la succession Beurard dressé le 5 septembre 1835 mentionne près d'un millier d'agates et de minéraux polies, un millier d'échantillons de minéraux et de roches, des cadres renfermant des insectes, des poissons et des coraux ainsi que quelques oiseaux empaillés ${ }^{22}$.

\section{Les «ichtyolites » de Münsterappel collectés par Beurard}

Les actinoptérygiens des dépôts permiens d'Allemagne sont connus depuis longtemps (Brignon, 2014). Les poissons du Kupferschiefer, littéralement «schiste cuivreux », d'Eisleben et de Mansfeld (bassin de la Saale au sud-est du massif du Harz) furent en effet signalés dès le XVI ${ }^{\mathrm{e}}$ siècle (Brandt, 1997 ; Gaudant et Bouillet, 1997, 2005). Martin Luther (1483-1546) faisait allusion en 1535 à ces poissons dans son «Genesisvorlesung» (Commentaire de la Genèse) (Dittmann, 2016: 535). D'autres écrivains et savants de la Renaissance mentionnaient les poissons des schistes cuivreux comme Giorgius Agricola (1494-1555), Conrad Gessner (1516-1565) et Bernard Palissy (vers 1510-vers 1590) (Agricola, 1546; Gessner, 1565: feuille 162; Palissy, 1580:130). Un de ces

\footnotetext{
${ }^{24}$ Né le 4 ventôse an 4 (23 février 1796) à Nancy (AD54, état civil, Nancy, naissances, 1795-1796, 5 Mi 394/R 112); mort le 10 novembre 1865 à Alger (ANOM, état civil, Alger, décès, 1865, acte $\mathrm{n}^{\mathrm{o}}$ 1036). Base Léonore, dossier LH/790/21.
} 
poissons servit même de modèle à la plus ancienne représentation d'un poisson fossile publiée dans la Cosmographie Universelle de Sebastian Münster (14881552) (Münster, 1550: 528). Les poissons dit «de Münsterappel », arrondissement du Mont-Tonnerre (Donnersberg), dans le bassin de Sarre-Nahe, ne furent quant à eux signalés qu'à la fin du XVIII ${ }^{\mathrm{e}}$ siècle (Dechen, 1848; Fournet, 1859 : 299; Heidtke, 2007). Ils proviennent principalement de la partie supérieure de la formation Meisenheim (M9) datée du Permien inférieur (Unter Rotliegend). Le mercure contenu dans ces dépôts de schistes bitumineux noirâtres entraîne la formation de cinabre à la surface des écailles des spécimens leur conférant des reflets rouges caractéristiques. Un des premiers à mentionner ces poissons et ce mode de fossilisation singulier fut Cosimo Alessandro Collini (1727-1806), directeur du cabinet d'histoire naturelle de Mannheim. Il écrivait à leur sujet: "Près de Moerschfeld, dans les mines de Mercure de Münster-Appell \& de Stein-Bockenheim on rencontre encore une particularité, dont je ne connois point d'exemple. Ce sont des poissons pétrifiés mercuriels. Ils sont dans l'intérieur d'un schiste noirâtre, \& pénétrés de taches \& de points de mine de Vif-argent rouge. On peut quelquefois séparer totalement leur corps de leur matrice. Ils sont si minces qu'ils n'ont que l'épaisseur d'un papier, ou d'un carton, \& sont si cassants \& si fragiles qu'il est fort difficile d'en avoir des entiers » (Collini, 1776: 44). La découverte de poissons fossiles dans les mines de mercure de Münsterappel fut également signalée dans plusieurs ouvrages et articles en allemand par le minéralogiste et géologue d'origine suédoise, Johann Jacob Ferber (1743-1790), le théologien et géologue d'origine suisse, Franz Cölestin von Beroldingen (1740-1798) et l'ingénieur des mines et naturaliste allemand Georg Adolf Suckow (1751-1813) (Ferber, 1776: 76-77; Beroldingen, 1778: 92, 1788: 199; Suckow, 1782: 351; Kölbel et al., 2002: 87).

Les poissons de Münsterappel appartiennent à l'espèce Paramblypterus duvernoy (Agassiz, 1833) (Actinopterygii, Amblypteridae) (Nottes et Heidtke, 1987 ; Dietze, 1999, 2001 ; Heidtke, 2007). L'espèce fut introduite de manière valide en 1833 par le paléoichtyologue suisse Louis Agassiz (18071873) dans la première livraison de ses Recherches sur les poissons fossiles sous le nom de Palaeoniscus [sic] ${ }^{25}$ duvernoy (Agassiz, 1833 : vol. 2, 45). Elle fut plus tard assignée au genre Paramblypterus Sauvage, 1888 (Boy, 1976; Štamberg, 2013, 2018). Il est à noter que les épithètes spécifiques «duvernoyi» et «duvernoi» introduites par différents auteurs (Huot, 1839: 467 ; Troschel, $1851: 520)$, puis largement reprises par la suite (Traquair, 1877 : 558; Woodward, 1891: 440; Boy, 1976; Dietze, 1999, 2001; Heidtke, 2007), constituent des orthographes subséquentes incorrectes et doivent être rejetées en vertu de l'article 33.3 du CINZ (ICZN, 1999). Agassiz introduisit également l'espèce Palaeoniscus minutus Agassiz, 1833 (dont l'orthographe correcte doit être Palaeoniscum minutum), pour un spécimen de petite taille provenant de Münsterappel (Agassiz, 1833: vol.2, 47) et correspondant

\footnotetext{
${ }^{25}$ L'orthographe originale Palaeoniscum Blainville, 1818a doit être retenue (Brignon, 2019b).
}

probablement à un juvénile de Paramblypterus duvernoy (Woodward, 1891: 441).

Dans son article sur les mines de mercure des nouveaux départements français de la rive gauche du Rhin, publié au mois de pluviôse an 6 (janvier-février 1798), Beurard faisait allusion aux poissons de Münsterappel sans toutefois avoir pu retrouver le gisement où ils avaient été découverts. Décrivant les mines de mercure de Münsterappel, Beurard (1798a: 322-323) déclarait qu' «on y a trouvé dans un temps, sur un schiste gris, des empreintes de poissons, pénétrées et mouchetées par l'oxide [sic] de mercure sulfuré rouge. Quoique je ne doute pas de ce fait, qui paraît trop bien constaté, je ne puis cependant me dispenser de déclarer,

$1^{\circ}$. Que je n'ai encore pu parvenir à voir aucune de ces sortes d'empreintes, quelques perquisitions que j'aie faites dans le pays;

$2^{\circ}$.Qu'ayant essayé, à mes frais, des recherches dans l'endroit où l'on m'a assuré qu'elles s'étaient trouvées, on ne $m$ 'a rapporté que des fragmens [sic] d'un schiste argileux, gris-foncé, chargé effectivement de dépouilles de poissons, mais sans aucun indice de cinabre: il est vrai que les eaux $n$ 'ont pas permis de pénétrer bien avant;

$3^{\circ}$. Que ce schiste mélangé de pareilles dépouilles ne se trouve pas précisément dans la montagne qui renferme la mine de mercure connue sous le nom de Münster-Apel [sic], mais dans une plus basse, qui lui est opposée, et dont elle est séparée par un vallon qu'arrose le ruisseau d'Apel [Appelbach], et que le village dit Münster-Apel remplit presque absolument à cet endroit. Les empreintes avec cinabre ont été rencontrées dans des travaux de recherches que l'on avait tentées de ce côté-là, et qui n'ont donné d'ailleurs aucun autre indice de minérai $[$ sic $] \gg$.

Beurard persévéra dans ses recherches et finit par redécouvrir enfin le fameux gisement de poissons fossiles de Münsterappel sur lesquels il publia le premier article leur étant exclusivement consacré (Fig. 3). Il précisait qu'il se trouvait «dans les flancs d'une montagne stratifiée, dont un coté porte le nom de Spreit, et l'opposé celui d'Himmelsberg», au sud-est de Münsterappel. Il notait que «ces dépouilles sont tellement comprimées, que les plus fortes ont à peine un millimètre d'épaisseur. L'animal est parfaitement reconnaissable en toutes ses parties, cependant les nageoires, la queue, les opercules des oreilles, et quelques autres portions de la tête, sont sensiblement plus marquées. Quoiqu'il soit en général assez aisé de les détacher, même quelquefois dans leur entier, cependant on ne peut guère les manier sans qu'elles ne se brisent. Dans quelques-unes la cassure est feuilletée, dans d'autres elle est en fragmens solides, indéterminés, à bords aigus et éclatans. Le mercure sulfuré se montre disséminé sur la surface, en filets ou traits déliés et courts, qui suivent et rendent plus sensibles les saillies ou raies des écailles, souvent en en dessinant parfaitement la forme; ou bien il est par taches superficielles informes qui se voient aussi sur les faces des feuillets intérieurs». Beurard avait également observé «des espèces de noyaux oblongs, renflés par le milieu, composés d'une substance bitumineuse d'un noir parfait, nuancé par une infinité de petites taches de mercure sulfuré d'un rouge violacé » qu'il pensait être des «musculites», autrement dit des sortes de moules pétrifiées. Il s'agit là probablement de coprolithes qui se rencontrent fréquemment dans la formation Meisenheim (Heidtke, 2007). 


\section{JOUR NAL DES MINES. \\ $\mathrm{N}^{\circ}$.84. FRUCTIDOR AN II. \\ SUR des Ichtyolives moucheiés de mercure sulfuré, trouvés dans le département du Mont-Tonnerre. \\ Par le Cit. Beurard, Agent du Gouvernement. \\ D \\ Eva craindre que ce que j'ai dit dans les observations générales qui préceddent quelques- uns de mes Rapports, insérés dans le Journal des Mines, pluviôse an 6, page 322, ait fát naître des doutes sur l'authenticité d'un fait rap- porté par plusieurs auteurs, qu'il asait été trouvé autrefois, dans les environs de Muns- terappel, des dépouilles et empreintes de pois- sons, mouchetées de mercure sulfuré; je me crois obligé aujourd'hui d'annoncer que la con- tinuation de mes recherches m'en a enfin pro- curé. \\ Je les ai rencontrées dans les flancs d'une montagne stratifiée, dont un côté porte le nom de Spreit, et l'opposé celui d'Himmelsberg; sa forme est celle d'un promontoire ou cap, qui s'avance entre deux vallons étroits vers un troisième plus large, et se confond avec une des chaînes du Mont-Tonnerre. \\ Volume 14. \\ $\mathrm{D} \mathrm{d}$}

Fig. 3. Page de titre de l'article de Beurard sur les «Ichtyolites mouchetés de mercure sulfuré» de Münsterappel publié en aoûtseptembre 1803 (fructidor an 11).

Fig. 3. Title page of Beurard's article on Münsterappel's "Ichtyolites mouchetés de mercure sulfuré" [Ichtyolites spotted with sulphurous mercury] published in August or September 1803.

L'ancien catalogue manuscrit des poissons fossiles conservés dans les collections de l'école des Mines de Paris permet de préciser la date à laquelle Beurard avait redécouvert les poissons de Münsterappel (Fig. 4) ${ }^{26}$. L'agent du

gouvernement avait en effet envoyé pour le cabinet de cette école quatre spécimens au mois de germinal de l'an 8 (marsavril 1800). La date de leur découverte est indiquée au 14 messidor an 7 (2 juillet 1799) dans le catalogue. Beurard communiqua également au Muséum d'Histoire naturelle, à Paris, plusieurs «ichtyolites» de Münsterappel comme le soulignait Barthélémy Faujas de Saint-Fond (1741-1819) et Henri Marie Ducrotay de Blainville (1777-1850), à qui l'on doit la première étude scientifique sur les poissons fossiles (Faujas de Saint-Fond, 1809: 349; Blainville, 1818a: 393, 1818b : 89; Brignon, 2016b). Dans sa description du Muséum parue en 1823 , Deleuze (1823: 344) mentionnait «un assez grand nombre de poissons dont le squelette est rempli de mercure sulfuré» et précisait qu'ils avaient été découverts et recueillis par Beurard dans les "grès houillers » des mines de mercure du Palatinat. Lors de son séjour à Paris entre décembre 1831 et juin 1832, Agassiz (1833: vol. 2, 45) avait vu au Muséum des spécimens de «Palaeoniscus Duvernoy» [sic] portant l'étiquette «Poissons fossiles pénétrés de mercure, renfermés dans un schiste bitumineux de la commune de Munster-Appel, dans le duché de Deux-Ponts ».

Aujourd'hui, les poissons du Permien de la région de Münsterappel ne sont pas rares dans les collections institutionnelles. Bien souvent, il est impossible de connaître le contexte historique de leur découverte en l'absence d'étiquette ou de détails consignés dans les registres anciens, même quand ces derniers existent encore. Les collections paléontologiques du MNHN conservent 5 spécimens de Paramblypterus duvernoy de Münsterappel. En particulier deux d'entre eux sont répertoriés dans l'inventaire des collections d'anatomie comparée rédigé en 1861 («Catalogue des ossements fossiles de vertébrés placés dans les galeries de géologie et minéralogie, volume deuxième», p. 1473). Ces spécimens dont les contre-empreintes sont également conservées portent les anciens numéros des collections d'anatomie comparée (initiales AC) : MNHN AC 10181, 10182, 10183 et 10184 (Fig. 5). Les étiquettes collées sur la face arrière des échantillons indiquent «Palaeoniscus Duvernoyi [...] $d u$ schiste bitumineux mercurial de Münster-Appel (terrain houiller)». Il est fort probable, sans toutefois qu'on puisse le démontrer, qu'ils faisaient partie des spécimens offerts par Beurard et mentionnés par Faujas de Saint-Fond, Deleuze et Agassiz. En revanche, un des poissons de Münsterappel collectés par Beurard de manière incontestable a pu être identifié dans les collections Géosciences de Sorbonne Université à Paris comme le prouve l'étiquette collée au verso du spécimen, écrite de la main même de Beurard (Fig. 6) : «Mercure sulfuré bituminifère pseudomorphique ou ichtyolite mouchetée de mercure sulfuré rouge écailleuse à la surface d'une argile schisteuse de Münsterapel [sic] département du Mont-Tonnere [sic], grande rareté sur laquelle j'ai donné une notice dans le journal des mines tome 14 page 409 $\left(n^{\circ} 84\right)^{27} \gg$.

\footnotetext{
$\overline{27}$ Il s'agit de la référence $B e u r a r d, 1803$.
}

\footnotetext{
${ }^{26}$ Ce catalogue est aujourd'hui conservé avec les collections de géologie de l'Université Lyon 1 où une partie des collections paléontologiques de l'école des Mines de Paris ont été transférées. Je remercie Emmanuel Robert de m'avoir fait connaître ce document.
} 


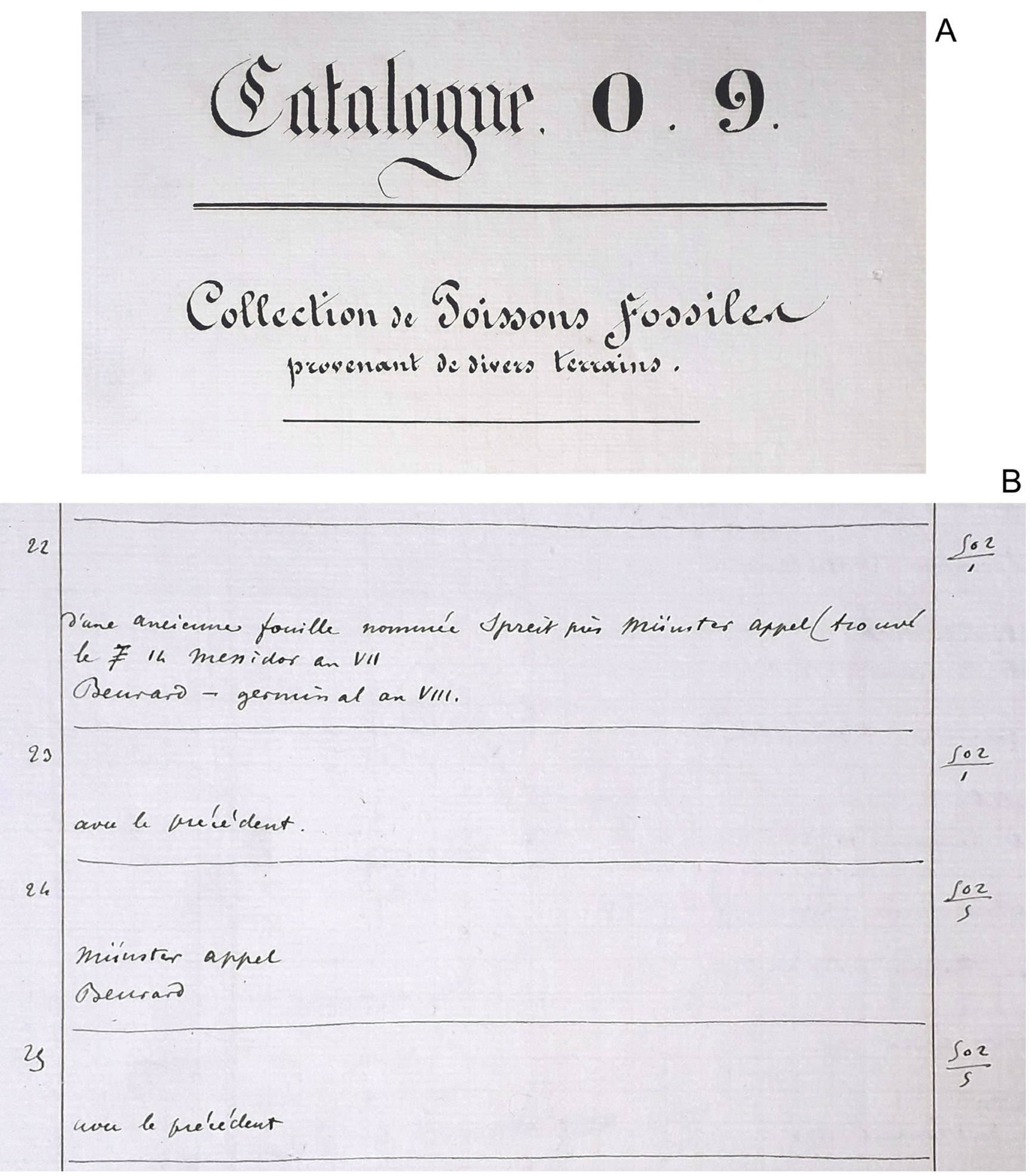

A

Fig. 4. Ancien catalogue manuscrit O.9 de l'école des Mines de Paris décrivant la «Collection de poissons fossiles provenant de divers terrains »; ce catalogue est conservé aujourd'hui dans les collections de géologie de l'Université Lyon 1; A : page de titre ; B : extrait relatif aux poissons de «Münster Appel» offerts par Beurard.

Fig. 4. Old manuscript catalogue O.9 of the Ecole des Mines de Paris [Paris School of Mines] describing the "Collection of fossil fish from various terrains"; this catalogue is now kept in the collections of geology of the Lyon 1 University; A: title page; B: extract relating to the fish from "Münster Appel” offered by Beurard. 


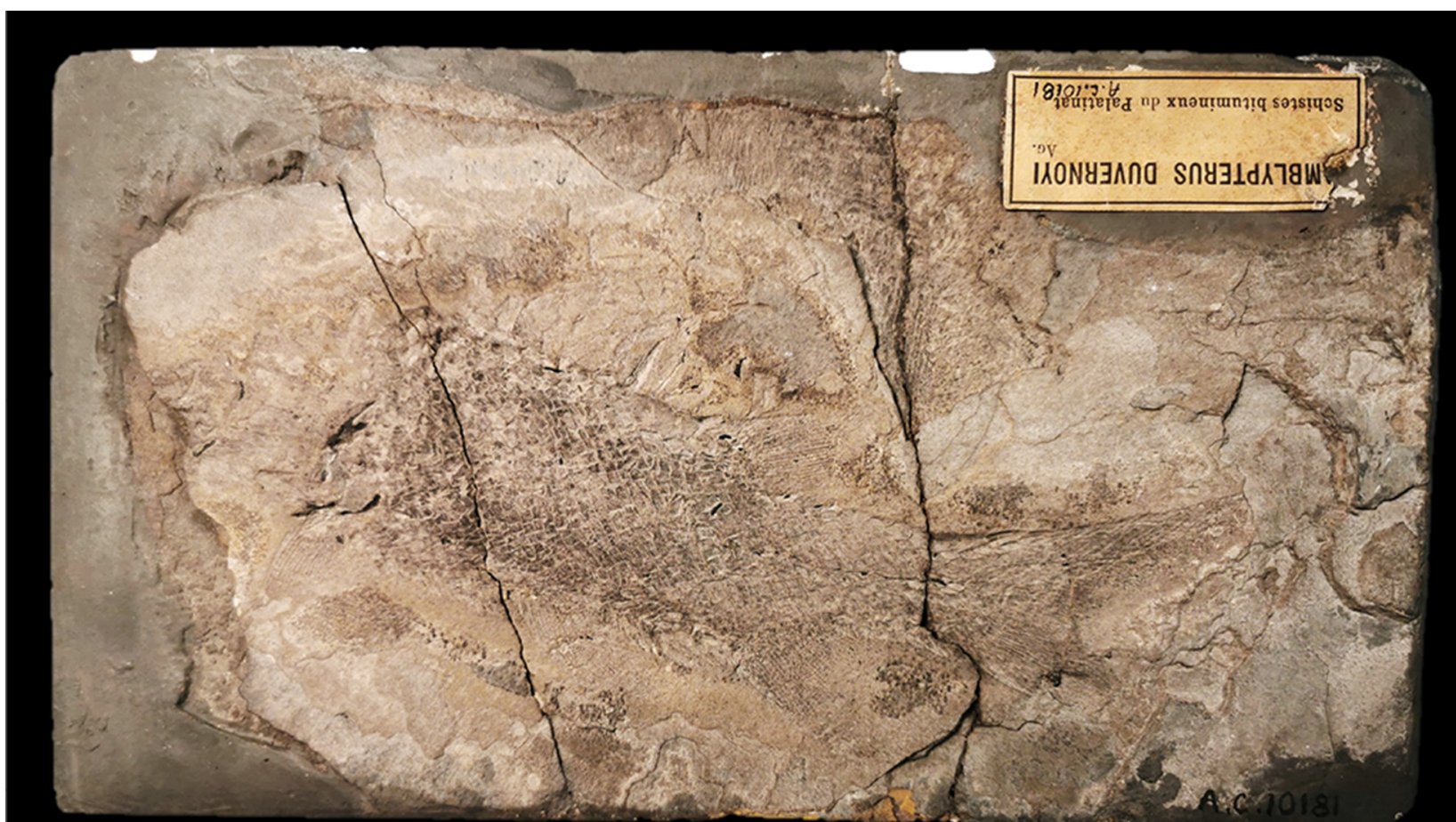

A

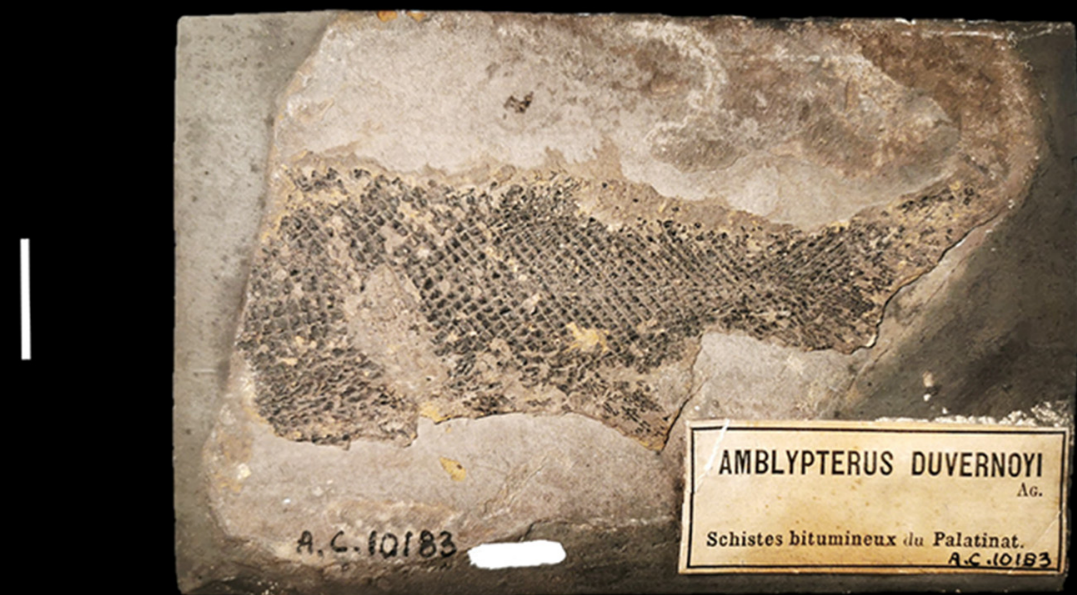

$B$
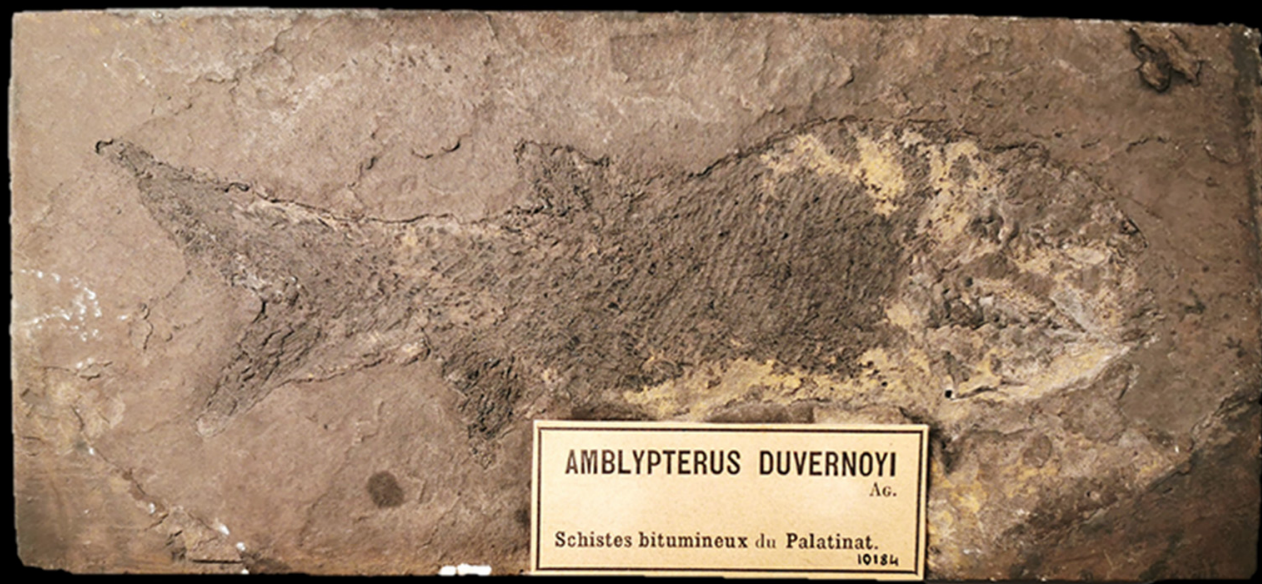

C

Fig. 5. Paramblypterus duvernoy (Agassiz, 1833), Formation Meisenheim, Permien inférieur, Münsterappel, anciennes collections d'anatomie comparée du MNHN. A : MNHN AC 10181. B : MNHN AC 10183 et sa contre-empreinte MNHN AC 10184 (C). Échelle : $2 \mathrm{~cm}$. Fig. 5. Paramblypterus duvernoy (Agassiz, 1833), Meisenheim Formation, Lower Permian, Münsterappel, former "anatomie comparée" [comparative anatomy] collections of the MNHN. A: MNHN AC 10181. B: MNHN AC 10183 with its counterpart MNHN AC 10184 (C). Scale bar: $2 \mathrm{~cm}$ 


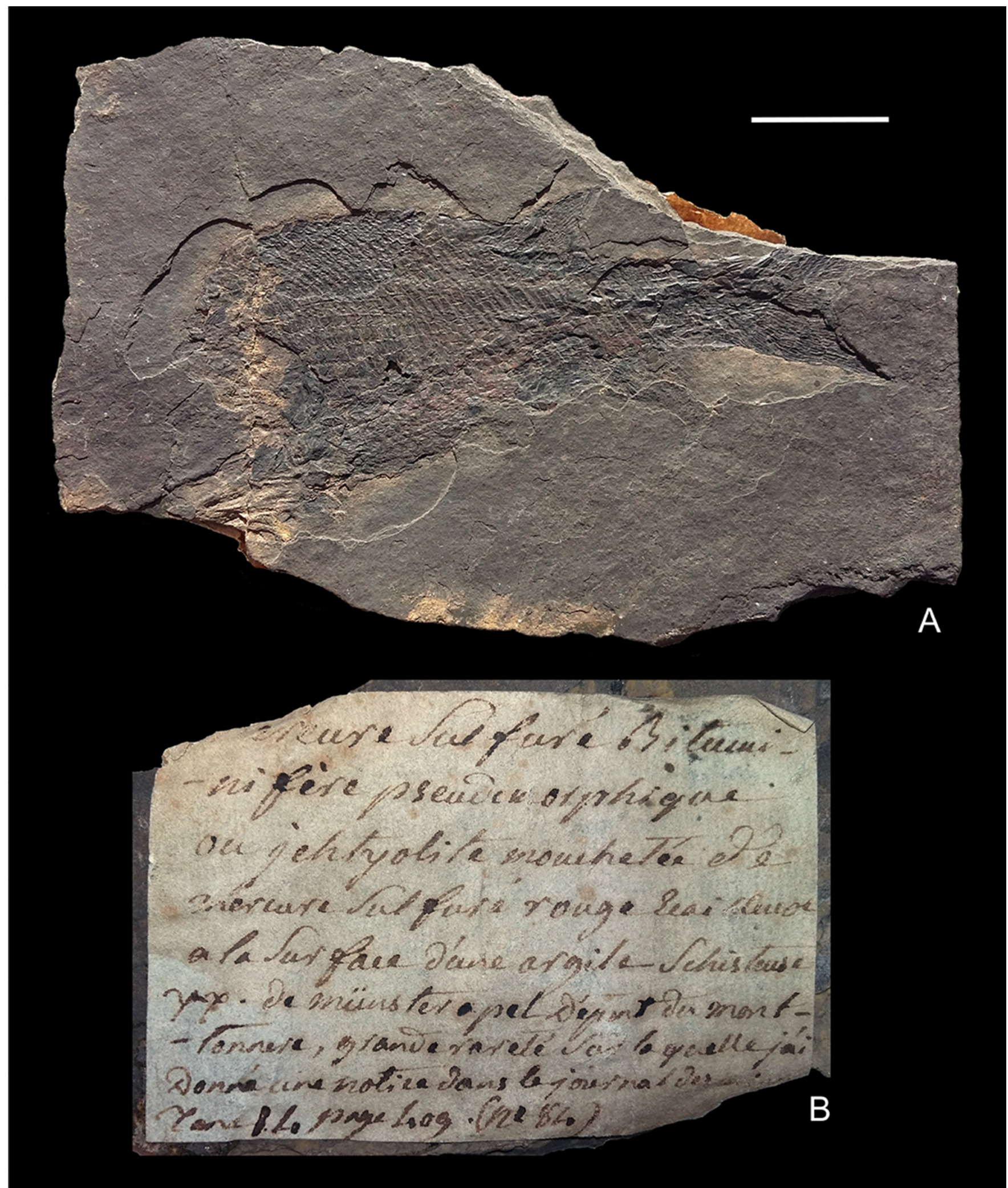

Fig. 6. A : Paramblypterus duvernoy (Agassiz, 1833), Formation Meisenheim, Permien inférieur, Münsterappel, collection Beurard, Sorbonne Université, SU.PAL.2018.0.86.1. B : Étiquette écrite par Beurard au dos du spécimen. Échelle : $2 \mathrm{~cm}$.

Fig. 6. A: Paramblypterus duvernoy (Agassiz, 1833), Meisenheim Formation, Lower Permian, Münsterappel, Beurard collection, Sorbonne Université, SU.PAL.2018.0.86.1. B: Handwritten label by Beurard affixed to the back of the specimen. Scale bar: $2 \mathrm{~cm}$. 

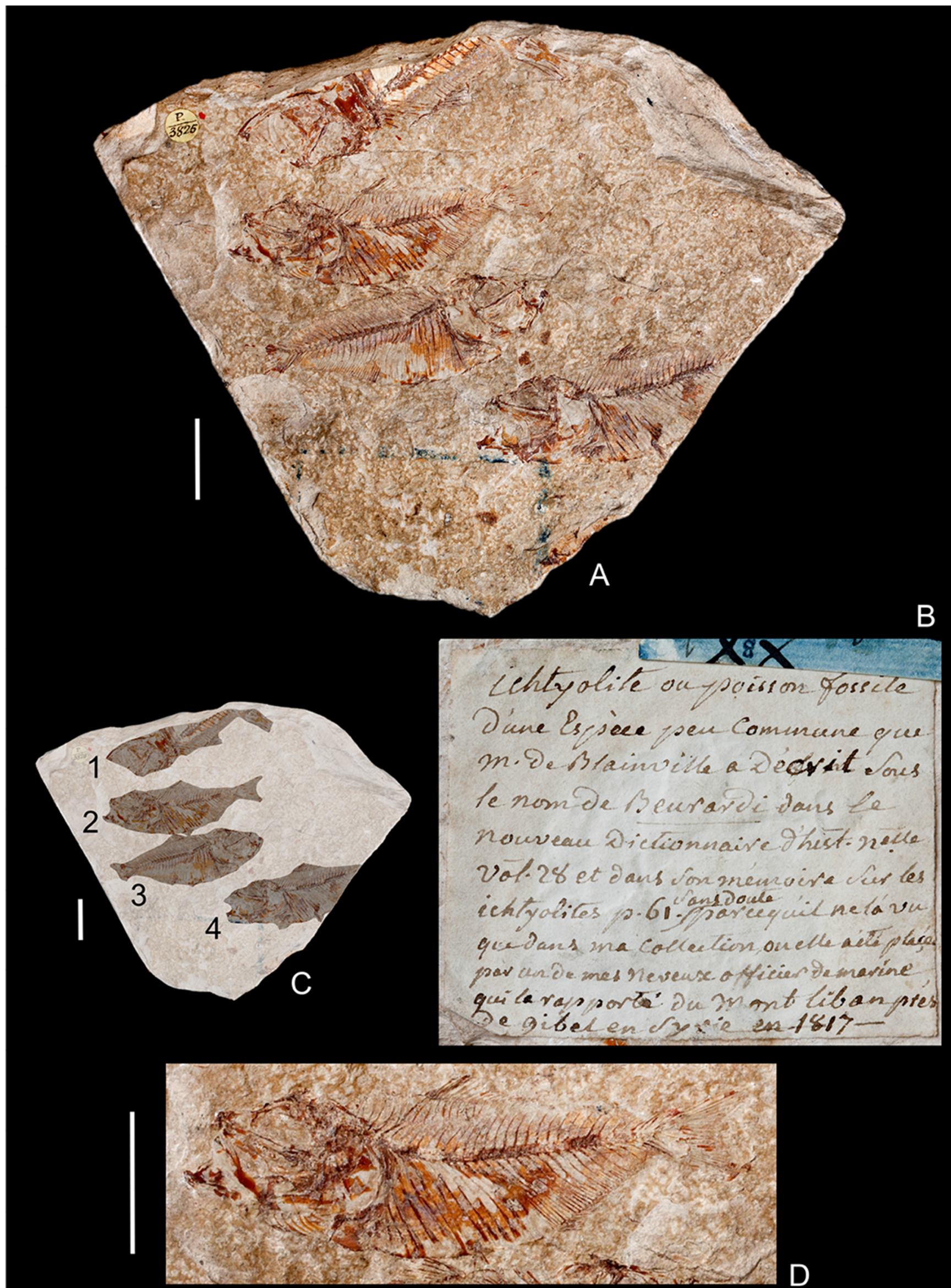

D 
Fig. 7. A : Armigatus brevissimus (Blainville, 1818a), série type, Cénomanien, Haqel, Liban, collections Beurard puis Enniskillen, NHMUK PV P3825. B : Étiquette écrite par Beurard collée au dos de l'échantillon. C: Vue schématique de l'échantillon mettant en évidence la désignation des spécimens par les chiffres 1 à 4. D : Vue agrandie du lectotype (spécimen 2) d'Armigatus brevissimus. Crédit photographique : (C) NHMUK, Londres. Échelles : $2 \mathrm{~cm}$.

Fig. 7. A: Armigatus brevissimus (Blainville, 1818a), type series, Cenomanian, Haqel, Lebanon, Beurard then Enniskillen collections, NHMUK PV P3825. B: Handwritten label by Beurard affixed to the back of the specimen block. C: Schematic view of the block with referred specimens labeled with numbers 1 to 4. D: Enlarged view of the here designated lectotype (specimen 2) of Armigatus brevissimus. Credit: () NHMUK, London. Scale bars: $2 \mathrm{~cm}$.

\section{Les poissons fossiles du Liban de la collection Beurard}

Blainville (1818a : 395, 1818b : 91) rapporte que Beurard possédait dans sa collection plusieurs poissons fossiles. Outre les poissons du Permien de Münsterappel, il avait fait l'acquisition de deux échantillons provenant du Mont Liban (Blainville, 1818a : 363-365, 1818b : 59-61). Ces poissons lui avaient été rapportés par son neveu Claude Charles Harmand, lieutenant de vaisseau, qui avait effectué une mission dans les mers du Levant du 4 janvier 1815 au 10 novembre 1817 à bord de la goélette la Biche. Mal orthographié «Armand» par Blainville (1818a: 364), Harmand naquit le 18 novembre 1784 à Nancy, paroisse Saint-Nicolas et mourut le 29 décembre 1847 dans cette même ville. Sa mère, Anne Charlotte Beurard (3 août 1754, Nancy, Saint-Sébastien - 22 juin 1842, Nancy), était une des sœurs de Jean-Baptiste Beurard. Son père, Joseph Harmand (10 mars 1750, Thiaucourt-Regniéville-16 mai 1836, Nancy), était avocat en parlement, secrétaire perpétuel de la chambre des consultations. Claude Charles Harmand fut reçu chevalier de l'ordre royal de la Légion d'honneur le 22 mai 1825 et chevalier de Saint-Louis ${ }^{28}$. Il fut également décoré de l'ordre des Deux-Siciles.

Les poissons fossiles du Crétacé du Liban sont connus au moins depuis le $\mathrm{IV}^{\mathrm{e}}$ siècle comme l'atteste les écrits d'Eusèbe de Césarée (vers 263-vers 339), évêque de Palestine, qui témoignait avoir vu différents genres de poissons de mer dans les pierres de construction exploitées dans les carrières sur les plus hautes montagnes du Liban (Gayet et al., 2012; Capasso, 2017). Dans son Livre des saintes paroles et des bons faiz de nostre saint roy Looÿs publié en 1547, Jean de Joinville, noble champenois et biographe de Saint-Louis relatait entre 1305 et 1309 que le roi au cours de la septième croisade (1248-1254) avait pu contempler lors de son séjour à Sidon, dans l'actuelle Liban, un poisson trouvé entre deux plaques de pierre. La première figure d'un poisson fossile du Liban fut publiée en 1698 par le voyageur et écrivain néerlandais Cornelis de Bruyn (1652-vers 1727) originaire de La Haye (Bruyn, 1698: fig. 158; Capasso, 2015). Au cours du dix-huitième siècle et au tout début du dix-neuvième siècle, plusieurs auteurs mentionnèrent également ces poissons (Maraldi, 1705; Scheuchzer, 1708: 25, 34-35, pl.5; Woodward, 1729: 21-22; Labat, 1735: 391-392; Guettard, 1783 : 427-431; Volney, 1787 : 273; Faujas de Saint-Fond, 1803: 118; Gayet et al., 2012).

Les deux échantillons de la collection Beurard furent découverts au "Mont-Liban, près de Gibel en Syrie», autrement dit près de Jbeil (Byblos), dans l'actuelle Liban. Il ne fait aucun doute qu'ils proviennent du célèbre gisement de Haqel (Pictet et Humbert, 1866: 70; Capasso, 2017), celui de

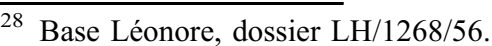

Hadjula n'ayant commencé à être exploité qu'à la fin du XIX siècle et au début du $\mathrm{XX}^{\mathrm{e}}$ siècle pour celui d'En Nammoura (Gayet et al., 2012). Les dépôts fossilifères de Haqel, comme ceux de Hadjula, seraient datés de la partie terminale du Cénomanien inférieur alors que ceux d'En Nammoura sont rattachés au Cénomanien moyen (Forey et al., 2003).

C'est à partir des deux échantillons de la collection Beurard que Blainville introduisit les deux premières espèces de poissons fossiles du Mont Liban utilisant la nomenclature binominale érigée par Linnaeus en 1758 (Gayet et al., 2012). Le premier d'entre eux servit à établir l'espèce Clupea brevissima Blainville, 1818a (Actinopterygii, Clupeomorpha). À noter que ce nom fut proposé avec l'orthographe «Clupaea brevissimus». Clupaea est une orthographe subséquente incorrecte du genre Clupea (Linnaeus, 1758). Par ailleurs, l'épithète «brevissimus» est une orthographe originale incorrecte et doit être corrigée en «brevissima » en vertu de l'article 34.2 du CINZ (ICZN, 1999). L'espèce fut assignée plus tard au genre Diplomystus Cope, 1877 (Woodward, 1888: 134, 1901 : 140) puis Armigatus Grande, 1982 dont elle est l'espèce type (Grande, 1982). Blainville précisait que le «morceau d'un décimètre carré» de la collection Beurard contenait «quatre squelettes » dont l'un d'eux avait une longueur de $75 \mathrm{~mm}$ et une hauteur de $22 \mathrm{~mm}$. Aucun type n'ayant été explicitement désigné parmi ces spécimens, ils constituent tous les quatre les syntypes d'Armigatus brevissimus (ICZN, 1999: article 72.1.1). Cette espèce est la plus abondante dans les gisements de Haqel et de Hadjula (Gayet et al., 2012). À partir du second échantillon de la collection Beurard, Blainville décrivit l'espèce Clupea beurardi Blainville, 1818a, en l'honneur de l'ancien agent du Gouvernement. Ce spécimen avait une longueur totale (TL) de $150 \mathrm{~mm}$ pour une hauteur(TD) de $20 \mathrm{~mm}$. Blainville rattachait également à cette espèce, quoiqu'avec doute, un spécimen de la collection de Barthélémy Faujas de Fond indiqué comme provenant de Saint-Jean-d'Acre (Acre, Israël). Ce dernier spécimen passa dans la collection de François Théophile Marie Régley (17771833 ) et fut décrit et figuré par Agassiz (1839: vol. 5, pl. 61, fig. $2 ; 1843$ : vol. $5,2^{\mathrm{e}}$ partie, 117).

Une partie de la collection de poissons fossiles de Beurard fut acquise par le célèbre collectionneur britannique, William Willoughby Cole (1807-1886), troisième comte d'Enniskillen, qui avait formé avec son ami Philip de Malpas Grey Egerton (1806-1881) les deux plus importantes collections paléoichtyologiques du $\mathrm{XIX}^{\mathrm{e}}$ siècle. Le catalogue de ces deux collections, dressé en 1836 et 1837 par Egerton (1836: 376, 1837), mentionne la présence d'un spécimen de l'espèce Clupea beurardi du Mont Liban. Louis Agassiz, qui étudia ces collections au cours de ses voyages en Grande-Bretagne, révèle que ce spécimen faisait bien partie de la collection Beurard et qu'il s'agissait même selon le paléontologue suisse du type à partir duquel Bainville avait établi Clupea beurardi. Le spécimen en question portait en effet une étiquette écrite de 
Tableau 1. Paramètres biométriques du lectotype (NHMUK PV P3825, spécimen 2) et d'un des paralectotypes (NHMUK PV P3825, spécimen 3) d'Armigatus brevissimus, mesures en mm, abréviations et définitions d'après Forey et al. (2003 : fig. 2); TL : longueur totale; SL : longueur standard ; HL : longueur de la tête; PD : longueur prédorsale; PV: longueur préventrale; PA : longueur préanale; TD : hauteur maximale du corps ; vert : nombre de vertèbres ; D.pt : nombre de ptérygophores dorsaux; A.pt : nombre de ptérygophores anaux ; s.na : nombre de supraneuraux ; ribs : nombre de paires de côtes pleurales. Les paramètres pour les autres spécimens d'Armigatus brevissimus et les autres espèces du genre Armigatus sont tirées des travaux de Forey et al. (2003), Murray et al. (2016), Vernygora et Murray (2016, 2021) et Alvarado-Ortega et al. (2020).

Table 1. Biometric parameters of the here designated lectotype (NHMUK PV P3825, specimen 2) and one of the paralectotype (NHMUK PV P3825, specimen 3) of Armigatus brevissimus, all linear measurements in mm, abbreviations and definitions after Forey et al. (2003: fig. 2); TL: total length; SL: standard length; HL: head length; PD: predorsal length; PV: preventral length; PA: preanal length; TD: maximum depth of body; vert: number of vertebrae; D.pt: number of dorsal pterygiophores; A.pt: number of anal pterygiophores; s.na: number of supraneurals; ribs: number of pairs of pleural ribs. Data for other specimens of Armigatus brevissimus and other species of Armigatus are from Forey et al. (2003), Murray et al. (2016), Vernygora and Murray (2016, 2021) and Alvarado-Ortega et al. (2020).

\begin{tabular}{|c|c|c|c|c|c|c|c|c|}
\hline & $\begin{array}{r}\text { A. bre } \\
\text { NHMUK } \\
\text { Spécimen } 2 \\
\text { Lectotype }\end{array}$ & $\begin{array}{l}\text { issimus } \\
\text { PV P3825 } \\
\text { Spécimen } 3 \\
\text { Paralectotype }\end{array}$ & $\begin{array}{l}\text { Armigatus } \\
\text { brevissimus } \\
\text { (autres spécimens) }\end{array}$ & $\begin{array}{l}\text { Armigatus } \\
\text { namouraensis }\end{array}$ & $\begin{array}{l}\text { Armigatus } \\
\text { alticorpus }\end{array}$ & $\begin{array}{l}\text { Armigatus } \\
\text { oligodentatus }\end{array}$ & $\begin{array}{l}\text { Armigatus } \\
\text { dalmaticus }\end{array}$ & $\begin{array}{l}\text { Armigatus } \\
\text { carrenoae }\end{array}$ \\
\hline TL & 75 & & & & & & & \\
\hline SL & 65 & 65 & & & & & & \\
\hline HL & 24 & 22 & & & & & & \\
\hline PV & 36 & 31 & & & & & & \\
\hline PA & 47 & 43 & & & & & & \\
\hline TD & 22 & 23 & & & & & & \\
\hline $\mathrm{HL} / \mathrm{SL}$ & 0,37 & 0,34 & $0,30-0,38$ & $0,28-0,35$ & $0,33-0,36$ & $0,31-0,35$ & $0,28-0,33$ & $0,36-0,4$ \\
\hline $\mathrm{PD} / \mathrm{SL}$ & 0,45 & 0,51 & $0,44-0,49$ & $0,43-0,52$ & $0,48-0,51$ & $0,46-0,52$ & $0,43-0,5$ & $0,55-0,58$ \\
\hline D.pt & 16 & & 17 & $15-17$ & $14-15$ & 13 & $13-14$ & $11-12$ \\
\hline A.pt & 28 & 28 & $26-29$ & $21-24$ & $26-27$ & $12-14$ & 17 & $15-17$ \\
\hline ribs & 13 & & $11-12$ & $16-18$ & 13 & $15-18$ & $16-19$ & $14-15$ \\
\hline
\end{tabular}

la main de Beurard dont Agassiz (1843 : vol. 5, $2^{\mathrm{e}}$ partie, 117) donnait la transcription suivante: «Ichthyolite ou poisson fossile d'une espèce peu commune, que M. de Blainville a décrit sous le nom de Beurardi, dans le nouveau Dictionnaire d'histoire naturelle [Blainville, 1818a] et dans son Mémoire sur les Ichthyolites, pag. 61 [Blainville, 1818b]; sans doute parce qu'il ne l'a vue que dans ma collection, où elle a été placée par un de mes neveux, officier de marine, qui l'a rapportée du Mont-Liban, près de Gibel en Syrie, en 1817».

Le British Museum fit l'acquisition de la collection Enniskillen en 1882 et 1883 (British Museum, 1904: 233234 ; Brignon, 2016b, 2019c). Le spécimen du Mont Liban de la collection Beurard acquis par Enniskillen est ainsi mentionné par Woodward (1901: 142) dans le Catalogue of the fossil Fishes in the British Museum sous le numéro P. $3825^{29}$. Woodward indique que les poissons visibles sur l'échantillon sont typiques de l'espèce «Diplomystus»

\footnotetext{
29 Plusieurs spécimens de Paramblypterus duvernoy de la région de Münsterappel sont conservés dans les collections Enniskillen et Egerton (1836, 1837 ; Woodward, 1891 : 442), aujourd'hui préservées au NHMUK (NHMUK PV P984, P985, P985a, P987, P3465, P3466, P4352). Aucun indice ne permet cependant d'affirmer qu'ils proviennent de l'ancienne collection de Beurard.
}

brevissimus (Blainville, 1818a), et qu'ils ne correspondent aucunement à la description qu'avait faite Blainville de l'espèce Clupea beurardi. Woodward concluait en conséquence qu'il fallait rejeter cette espèce en raison du fait que ces prétendus spécimens types ne correspondaient pas à la description originale de Blainville.

L'échantillon en question est toujours conservé au Natural History Museum à Londres (Fig. 7). Il porte bien l'étiquette manuscrite que Beurard avait écrite et qu'Agassiz avait transcrite en 1843. Sur sa face principale, la plaque porte quatre spécimens dont le plus complet (spécimen 2 sur la Fig. 7) possède une longueur totale (TL) de $75 \mathrm{~mm}$ et une hauteur maximale (TD) de $22 \mathrm{~mm}$. Ils correspondent en réalité exactement à la description de ceux à partir desquels Blainville fonda Clupea brevissima. Beurard avait donc placé par erreur une étiquette indiquant «Clupea beurardi» sur l'échantillon portant la série type de Clupea brevissima. Cette erreur, que n'avait pas décelée Agassiz (1843: vol. 5, 2 ${ }^{\mathrm{e}}$ partie, 117) ni Davis (1887 : 576-577) n'avait pas été complètement démêlée par Woodward. Ce dernier concluait à juste titre que les spécimens de l'échantillon P. 3825 ne correspondaient pas à la description de Clupea beurardi faite par Blainville et qu'ils étaient en revanche typiques de «Diplomystus» brevissimus. Et pour cause, les quatre spécimens de l'échantillon P. 3825, 
qui porte aujourd'hui le numéro d'inventaire NHMUK PV P3825, ne sont autres que les syntypes d'Armigatus brevissimus (Blainville, 1818a) qui étaient restés totalement inaperçus jusqu'à maintenant.

Le spécimen de la collection Beurard désigné sous le nom de Clupea beurardi par Blainville n'a quant à lui pas pu être retrouvé. Le spécimen de l'ancienne collection Faujas de Saint-Fond figuré par Agassiz n'étant rattaché qu'avec doute par Blainville à cette espèce, seule le spécimen de la collection Beurard peut être considéré comme son spécimen porte nom, ce qui lui confère le statut d'holotype par monotypie de Clupea beurardi. Tant que ce spécimen reste perdu, cette espèce doit être considérée comme un nomen dubium, la description seule donnée par Blainville ne permettant pas d'en donner une diagnose suffisante.

\section{Paléontologie systématique}

Subdivision: TELEOSTEI Müller, 1845

Superordre: CLUPEOMORPHA Greenwood et al., 1966 Ordre : ELLIMMICHTHYIFORMES Grande, 1985

Famille: Armigatidae Murray et Wilson, 2013

Genre: Armigatus Grande, 1982

Diagnose : voir Grande (1982: 4) et Forey et al. (2003: 275-276)

Espèce type par désignation originale (Grande, 1982): Clupea brevissima Blainville, 1818a (nom introduit par Blainville avec l'orthographe incorrecte Clupaea brevissimus).

Armigatus brevissimus (Blainville, 1818a)

Matériel type : NHMUK PV P3825, quatre spécimens sur une même plaque apportée en France en 1817 par Claude Charles Harmand (1784-1847) et conservée dans la collection Beurard à Paris puis celle de Lord Enniskillen en Irlande du Nord, avant son acquisition par le British Museum (Natural History) à Londres (Fig. 7A-7C). Décris par Blainville (1818a: 364-365, 1818b: 60-61) et en l'absence de désignation d'un type par ce dernier, ces quatre spécimens constituent les syntypes d'Armigatus brevissimus (Blainville, 1818a). Sur l'échantillon NHMUK PV P3825, le spécimen 1 est très mal conservé et indéterminable. La partie postérieure du spécimen 4 et l'extrémité postérieure de la nageoire caudale du spécimen 3 sont manquantes. Le spécimen 2 (Fig. 7D) est le plus complet et permet l'analyse biomètrique la plus précise (Tab. 1). Il est en conséquence explicitement désigné ici lectotype d'Armigatus brevissimus afin de fixer les caractéristiques de l'espèce (ICZN, 1999: article 74.7). Les spécimens 1,3 et 4 deviennent donc les paralectotypes d'Armigatus brevissimus.

Localité et âge types: Haqel (Liban, Moyen-Orient), Cénomanien.

Discussion : les paramètres biomètriques du lectotype correspondent à ceux des spécimens du Cénomanien de Haqel classiquement assignés à cette espèce (voir par exemple Forey et al., 2003 : tab. 9). Il correspond à un poisson clupéomorphe relativement allongé avec un rapport TD/SL de 0,34 (voir Tab. 1 la définition des abréviations), ce qui le distingue facilement d'Armigatus alticorpus Forey et al., 2003 du Cénomanien d'En Nammoura et Haqel, possédant un rapport TD/SL compris typiquement entre 0,46 et 0,53 (Forey et al., 2003; Vernygora et Murray, 2021). Il possède 6 supraneuraux et 16 ptérygophores dorsaux. Ses 28 ptérygophores anaux supportant les rayons de la nageoire anale permettent de le distinguer d'Armigatus namouraensis Forey et al., 2003 du Cénomanien moyen d'En Nammoura (Liban), d'Armigatus dalmaticus Murray et al., 2016 du Sénonien (probablement Campanien) de Croatie, d'Armigatus carrenoae Alvarado-Ortega et al., 2020 de l'Albien du Méxique et d'Armigatus oligodentatus Vernygora et Murray, 2016, du Cénomanien/Turonien du Maroc qui n'en possèdent respectivement que 21-24, 17, 1517 et 12-14 (Forey et al., 2003; Murray et al., 2016; Vernygora et Murray, 2016; Alvarado-Ortega et al., 2020). Le lectotype d'A. brevissimus possède 31 vertèbres contre 35-37 pour A. alticorpus, 36-38 pour A.namouraensis, 32-37 pour A. dalmaticus, 33-35 pour d'A. carrenoae et 37-39 pour A. oligodentatus. Son rapport TD/SL, ses relatifs grand nombre de ptérygophores et de rayons à sa nageoire anale et faibles nombres de vertèbres et de paires de côtes pleurales permettent donc de distinguer $A$. brevissimus de toutes les autres espèces connues du genre Armigatus (Tab. 1).

\section{Conclusion}

Mal connu dans l'histoire des sciences, Jean-Baptiste Beurard a pourtant joué un rôle indéniable dans les débuts de la paléoichtyologie française. Il a notamment contribué à rendre célèbre le gisement permien de Münsterappel et ses poissons amblyptérides recouverts de cinabre. Lui-même collectionneur de minéraux et de fossiles, il avait constitué une collection de poissons fossiles qui faisait partie de la petite dizaine de collections connues à Paris à la fin des années 1810 , comme celles d'Étienne de Drée, Alexandre Brongniart (1770-1847), Barthélémy Faujas de Saint-Fond, François Théophile Marie Régley, Francois Pierre Nicolas Gillet de Laumont, François Jean-Baptiste Ménard de la Groye (1775-1827), Anselme Gaëtan Desmarest (1784-1838) et Dominique-Sébastien Léman (1781-1829) (Blainville, 1818a: 395, 1818b: 91). La collection Beurard fut utilisée par Henri Marie Ducrotay de Blainville pour une des premières études scientifiques exclusivement consacrée aux «poissons » fossiles (Chondrichthyes et Osteichthyes) dans laquelle ce dernier décrit en utilisant la nomenclature binominale près de 150 espèces, dont plus d'une soixantaine sont nouvelles. À partir du matériel de cette collection, Blainville décrivit la première espèce valide de téléostéen du célèbre gisement cénomanien de Haqel au Liban, l'emblématique clupéomorphe Armigatus brevissimus, dont la série type a pu être redécouverte.

\section{Abréviations}

AD54 Archives départementales de Meurthe-et-Moselle, Nancy

AD55 Archives départementales de la Meuse, Bar-le-Duc

AD75 Archives de l'état civil reconstitué de Paris

AN Archives nationales, Paris et Pierrefitte-sur-Seine

ANOM Archives nationales d'outre-mer

CINZ Code international de nomenclature zoologique

ICZN International Commission on Zoological Nomenclature

SU Collections Géosciences, Sorbonne Université, Paris 
Remerciements. J'adresse mes vifs remerciements à Emma Bernard (NHMUK) pour l'envoi de photographies de spécimens. Je tiens également à exprimer toute ma gratitude à Stéphane Jouve (SU), Alan Pradel (MNHN), Emmanuel Robert (Université Lyon 1) et Isabelle Rouget (SU) pour leur accueil dans les collections dont ils ont la charge. Je remercie les Archives nationales (sites de Pierrefitte-sur-Seine et de Paris) ainsi que le service du patrimoine des Archives départementales de la Meuse. Je remercie enfin Louis Taverne et un rapporteur anonyme pour leurs remarques qui ont permis d'améliorer le manuscrit.

\section{Références}

Agassiz J-L-R. 1833. Recherches sur les poissons fossiles, $1^{\text {re }}$ livraison. Neuchâtel: Petitpierre et Prince (texte) et $\mathrm{H}$. Nicolet (planches), vol. 1 : [i]-xii, [1]-16, pls A-G; vol. 2: [1]-48, pls A-C, 1-7; vol. 4 : 17-32, pls A, 1, 2; vol. 5, $1^{\text {re }}$ partie : 17-24, pls A, 1, 2.

Agassiz J-L-R. 1839. Recherches sur les poissons fossiles, $13^{\mathrm{e}}$ livraison. Neuchâtel: Petitpierre (texte) et H. Nicolet (planches), vol.2: pl. 69b; vol. 4 : 109-204, pl. 15-17, 26-36, 37a, 39, 41, 41a, 44; vol. 5 : pl. 15, 26, 30, 31, 43, 61 ; feuilleton additionnel : 127-130.

Agassiz J-L-R. 1843. Recherches sur les poissons fossiles, $17^{\mathrm{e}}$ livraison. Soleure et Neuchâtel: Jent et Gassmann (texte) et H. Nicolet (planches), vol. 2 : [i]-xii, 263-310; vol. 2, $2^{\mathrm{e}}$ partie : [i]-[ii], 73-336, [337]-[338], pl. E, H, 18, 23e, 65a ; vol. 3 : pl. A, J-M,M', M", N-Q, 36, 37, 37a; vol. 5 : [i]-xii, 1-16; vol. 5, $1^{\mathrm{e}}$ partie : $16 \mathrm{a}-$ 16h, 33-122; vol. 5, $2^{\mathrm{e}}$ partie : $85-160$, pl. M.

Agricola G. 1546. De ortu \& causis subterraneorum, lib. V. De natura eorum quæ effluunt ex terra, lib. IIII. De natura fossilium, lib. X. De ueteribus \& nouis metallis, lib. II. Bermannus, siue De re metallica dialogus. Interpretatio Germanica uocum rei metallicæ, addito Indice fœcundissimo. Basiliæ: Froben, $487+[52] \mathrm{p}$.

Alvarado-Ortega J, Than-Marchese BA, Melgarejo-Damián MP. 2020. On the Albian occurrence of Armigatus (Teleostei, Clupeomorpha) in America, a new species from the Tlayúa Lagerstätte, Mexico. Palaeontologia Electronica 23(3): a52.

Anonyme. 1805. Analyse des travaux de l'Académie des sciences, arts et belles-lettres de Dijon, pendant le cours de l'an 12. In: Séance Publique de l'Académie des Sciences, Arts et Belles-Lettres de Dijon, an 13, pp. 1-72.

Anonyme. 1809. Gelehrte Gesellschaften und Preise. Intelligenzblatt der Jenaischen Allgemeine Literatur-Zeitung 35: 299-302.

Antoine J-B, Toussaint M. 1836. Liste des membres de l'Académie des Sciences, Arts et Belles-Lettres de Dijon, pour l'année 1836. Mémoires de l'Académie des Sciences, Arts et Belles-Lettres de Dijon, année 1836: 163-179.

Aulard F-A. 1910. Recueil des actes du Comité de salut public avec la correspondance officielle des représentants en mission et le registre $\mathrm{du}$ Conseil exécutif provisoire, tome 20. Paris: Imprimerie nationale, [iv] $+839 \mathrm{p}$.

Becquart N, Colnat J. 1958. Archives départementales de la Meuse. Inventaire sommaire de la série $\mathrm{H}$ (Clergé régulier), tome 1. Bar-leDuc : Imprimerie Jolibois, [ii] $+547 \mathrm{p}$.

Benoît A. 1884. Les bibliophiles, les collectionneurs et les bibliothèques des monastères des trois évêchés (1552-1790). Nancy et Bruxelles : R. Wiener et R. Dupriez, xvi + 300 + [4] p.

Bernard EL, Smith M. 2015. Arthur Smith Woodward's fossil fish type specimens. Geological Society, Special Publications 430: 87-88.

Beroldingen FC von. 1778. Beobachtungen, Zweifel, und Fragen die Mineralogie überhaupt, und insbesondere ein natürliches MineralSystem betreffend. Erster Versuch. Hannover: Johann Wilhelm Schmidt, [viii] $+203+[1] \mathrm{p}$.
Beroldingen $\mathrm{FC}$ von. 1788. Bemerkungen auf einer Reise durch die Pfälzischen und Zweybrückschen Quecksilber-Bergwerke. Berlin: Friedrich Nicolai, [xvi] $+240 \mathrm{p}$.

Beurard J-B. 1797. Rapport sur la mine de cuivre de Fischbach. Journal des Mines 6(34): 797-804.

Beurard J-B. 1798a. Rapports sur quelques mines de mercure situées dans les nouveaux départemens de la rive gauche du Rhin. Journal des Mines 7(41): 321-360.

Beurard J-B. 1798b. Rapport abrégé sur les mines de houille des environs de Meisenheim, ci-devant pays de Deux-Ponts. Journal des Mines 8(44): 609-614.

Beurard J-B. 1803. Notice sur des ichtyolites mouchetés de mercure sulfuré, trouvés dans le département du Mont-Tonnerre. Journal des Mines 14(84): 409-414.

Beurard J-B. 1809. Dictionnaire allemand-français contenant les termes propres à l'exploitation des mines, à la minéralurgie et à la minéralogie. Paris : Imprimerie de Madame Huzard, 696 p.

Beurard J-B. 1814a. Notice sur les houillières de Borgloh. Journal des Mines 36(211): 63-72.

Beurard J-B. 1814b. Précis sur la saline de Lunebourg. Journal des Mines 36(214): 283-312.

Beurard J-B. 1814c. Extrait d'un rapport sur la saline de Rothenfeld. Journal des Mines 36(216): 445-458.

Beurard J-B. 1815. Extrait d'un mémoire sur les méthodes de distillation de mercure qui ont été et sont encore en usage dans le Palatinat. Journal des Mines 38(228): 401-414.

Beurard J-B. 1819a. Dictionnaire allemand-français contenant les termes propres à l'exploitation des mines, à la minéralurgie et à la minéralogie. Paris: Chez Mongie jeune, 696p.

Beurard J-B. 1819b. Précis sur la ville de Hambourg. Mémoires de la Société royale d'Arras, pour l'encouragement des Sciences, des Lettres et des Arts 2: 429-456.

Blainville HMD de. 1818a. Poissons fossiles. In : Nouveau Dictionnaire d'Histoire Naturelle appliquée aux arts, à l'agriculture, à l'économie rurale et domestique, à la médecine, etc. par une société de naturalistes et d'agriculteurs, tome 27, (Pla-Por). Paris : Deterville, pp. 310-395.

Blainville HMD de. 1818b. Sur les ichthyolites ou les poissons fossiles. Paris : extrait du Nouveau Dictionnaire d'Histoire Naturelle, $91 \mathrm{p}$.

Boy JA. 1976. Überblick über die Fauna des saarpfälzischen Rotliegenden (Unter-Perm). Mainzer Geowissenschaftliche Mitteilungen 5: 13-85.

Brandt S. 1997. Geschichten und Aberglauben I. Deutungen von Kupferschieferfossilien aus vergangener Zeit. Verein Mansfelder Berg-und Hüttenleute, Mitteilung 29(5): 2-7.

Brignon A. 2014. Les recherches paléoichthyologiques et géologiques sur le gisement permien de Muse près d'Autun (Saône-etLoire) au début du XIX ${ }^{\mathrm{e}}$ siècle. Bulletin de la Société Géologique de France 185(4): 233-252.

Brignon A. 2015. Faujas de Saint-Fond, Reinwardt, Cuvier et les poissons fossiles du Crétacé de la «Montagne Saint-Pierre» de Maastricht (Pays-Bas). Geodiversitas 37(1): 59-77.

Brignon A. 2016a. Redécouverte des «crocodiles fossiles des environs du Mans » de Georges Cuvier après deux siècles d'oubli. Bulletin de la Société Géologique de France 187(2): 105-120.

Brignon A. 2016b. Revue historique des premières études sur les poissons fossiles (Teleostei) des ardoises d'Engi (Oligocène inférieur, Canton de Glaris). Revue de Paléobiologie 35(2): 459-490.

Brignon A. 2017a. Les «ossements pétrifiés» du Jurassique des Vaches Noires dans les cabinets d'histoire naturelle du XVIII et du début du XIX ${ }^{\mathrm{e}}$ siècles. L'Écho des Falaises 21: 7-35.

Brignon A. 2017b. La collecte des vertébrés fossiles au Mont-Aimé (Marne) par le baron Charles-Louis de Ponsort (1792-1854). 
Bulletin d'Information des Géologues du Bassin de Paris 54(3): 20-44.

Brignon A. 2019a. Les conditions d'acquisition de la collection Gazola de poissons fossiles du Monte Bolca (Eocène, Italie) par le Muséum national d'Histoire naturelle. Geodiversitas 41(2): 11-123.

Brignon A. 2019b. The nomenclatural status of Palaeothrissum inaequilobum Blainville, 1818, P. parvum Blainville, 1818 and Aeduella blainvillei (Agassiz, 1833) (Actinopterygii, Aeduellidae). Carnets de Géologie 19(8): 141-148.

Brignon A. 2019c. Le diodon devenu requin : l'histoire des premières découvertes du genre Ptychodus (Chondrichthyes). Edité par l'auteur, Bourg-la-Reine, $100 \mathrm{p}$.

Brignon A. 2020. Les premières découvertes de vertébrés jurassiques aux Vaches Noires (Calvados, France). In: Actes du premier colloque de l'APVSM, "Paléontologie et Archéologie en Normandie», 5-6 octobre 2019. Bulletin de l'Association paléontologique de Villers-sur-Mer 2020: 7-40.

British Museum (Natural History). 1904. The history of the collections contained in the Natural History Departments of the British Museum, vol. 1. Londres: Printed by order of the Trustees of the British Museum, xvii $+442 \mathrm{p}$.

Bruyn C de. 1698. Reizen van Cornelis de Bruyn, door de vermaardste deelen van Klein Asia, de eylanden Scio, Rhodus, Cyprus, Metelino, Stanchio, \&c. Mitsgaders de voornaamste steden van Aegypten, Syrien en Palestina. Delft: Henrik van Krooneveld, $[18]+398+[8]$ p., 1 frontispice, 1 portrait, $104 \mathrm{pl}$.

Capasso L. 2014. The history of the fossil fish private collecting. Bollettino del Museo Civico di Storia Naturale di Verona 38 (Geologia Paleontologia Preistoria): 51-89.

Capasso L. 2015. The history of specimens collecting demonstrates the existence of stratigraphic variations in the Late Cretaceous fish fauna composition at Haqel, Lebanon. Bollettino del Museo Civico di Storia Naturale di Verona 39 (Geologia Paleontologia Preistoria): 47-54

Capasso L. 2017. The history and the situation of the world famous fossil fish quarries in Lebanon. Bollettino del Museo Civico di Storia Naturale di Verona 41 (Geologia Paleontologia Preistoria): 53-76.

Collini CA. 1776. Journal d'un voyage, qui contient différentes observations minéralogiques; particulièrement sur les agates, et le basalte. Avec un détail sur la manière de travailler les agates. Mannheim : C.F. Schwan, xii +384 p.

Cope ED. 1877. A contribution to the knowledge of the ichthyological fauna of the Green River shales. Bulletin of the United States Geological and Geographical Survey of the Territories 3(4): 807-819.

Davies GL. 1970. The palaeontological collection of Lord Cole, third Earl of Enniskillen (1807-1886), at Florence Court, Co. Fermanagh. The Irish Naturalists' Journal 16(12): 379-381.

Davis JW. 1887. The fossil fishes of the Chalk of Mount Lebanon in Syria. The Scientific Transaction of the Royal Dublin Society Series 2(3): 457-636, pl. 14-38.

Dechen $\mathrm{H}$ von. 1848. Das Vorkommen der Quecksilbererze in dem Pfälzisch-Saarbrückenschen Kohlen-Gebirge. Archiv für Mineralogie, Geognosie, Bergbau und Hüttenkunde 22(2): 375-464.

Deleuze JPF. 1823. Histoire et description du Muséum Royal d'Histoire Naturelle. Paris : Chez M.A. Royer, tome 1 : vi + 1-330, 3 plans, 5 pl.; tome 2 : [iv] $+331-720,9$ pl.

Dettmer H-G. 2015. Héron de Villefosse-Napoleons Mann im Harzer Revier. Harz-Zeitschrift 67: 94-105.

Dietze K. 1999. Paramblypterus duvernoyi (Actinopterygii): skull morphology and intra-specific variation, and its implications for the systematics of paramblypterid fishes. Journal of Vertebrate Paleontology 19(2): 247-262.
Dietze K. 2001. Biological aspects of an interesting fossil fish: Paramblypterus duvernoyi (Amblypteridae, Actinopterygii). Mitteilungen aus dem Museum für Naturkunde Berlin, Geowissenschaftliche Reihe 4: 121-138.

Dittmann R. 2016. Naturkenntnis und Kunstschaffen. Die Discours admirables von Bernard Palissy. Übersetzung und Kommentar. Berlin \& Boston: Walter De Gruyter GmbH, $587 \mathrm{p}$.

Durozoir C. 1843. Beurard (Jean-Baptiste). In: Biographie universelle ancienne et moderne, nouvelle édition, tome 4. Paris: A. Thoisnier Desplaces, p. 242.

Egerton PMG. 1836. Catalogue of fossil fish in the collections of Lord Cole and Sir Philip Grey Egerton, arranged alphabetically; with references to the localities, geological positions, and published descriptions of the species. The London and Edinburgh Philosophical Magazine and Journal of Science 8(48): 366-373.

Egerton PMG. 1837. A systematic and stratigraphical catalogue of the fossil fish in the cabinets of Lord Cole and Sir Philip Grey Egerton; together with an alphabetical and stratigraphical catalogue of the same species, with references to their published figures and descriptions. London: Richard and John E. Taylor, 24 p.

Engerand L. 1920. Principaux documents des Archives nationales relatifs aux régions de la Sarre et de la rive gauche du Rhin. Deuxième rapport fait au nom de la commission chargée d'examiner le projet de loi créant un office des mines domaniales de la Sarre (inventaire analytique F/14/1065-F/14/1087/2). Pierrefitte-sur-Seine: Archives nationales, $123 \mathrm{p}$.

Faujas de Saint-Fond B. 1803. Essai de Géologie, ou Mémoires pour servir à l'histoire naturelle du globe, tome 1. Paris : C.F. Patris, [iv] + 492 p. (1-400 et 403-[494]), 18 pls.

Faujas de Saint-Fond B. 1809. Essai de Géologie, ou Mémoires pour servir à l'histoire naturelle du globe, tome $2,1^{\mathrm{e}}$ partie. Paris : Gabriel Dufour et compagnie, [iv] $+400+[2]$ p., pl. 18-21, 20bis.

Ferber JJ. 1776. Bergmännische Nachrichten von den merkwürdigsten mineralischen Gegenden der Herzoglich-Zweybrückischen, Chur-Pfälzischen, Wild- und Rheingräflichen und Nassauischen Länder. Mietau: Jacob Friedrich Hinz, [xvi] +94 p.

Forey PL, Yi L, Patterson C, Davies CE. 2003. Fossil fishes from the Cenomanian (Upper Cretaceous) of Namoura, Lebanon. Journal of Systematic Palaeontology 1(4): 227-330.

Fournet J. 1859. Recherches sur la constitution géologique des montagnes du Palatinat du Rhin et sur la formation des spilites agatifères. Mémoires de l'Académie impériale des Sciences, BellesLettres et Arts de Lyon, Classe des Sciences 9: 287-330.

Gaudant J, Bouillet G. 1997. Aux sources de la paléoichthyologie : les doléances et revendications des poissons (Piscium querelae et vindiciae) de Johann Jakob Scheuchzer (1708). In : Gaudant J, Gohau $\mathrm{G}$, eds. De la géologie à son histoire. Paris : CTHS, pp. 37-59.

Gaudant J, Bouillet G. 2005. La paléontologie de la Renaissance. Travaux du Comité Français d'Histoire de la Géologie série 3, 19 (3): $35-50$.

Gayet M, Abi Saad P, Gaudant O. 2012. Les fossiles du Liban, mémoire du temps. Gap : Éditions Désiris, $184 \mathrm{p}$.

Gessner C. 1565. De Rerum fossilium lapidum et gemmarum maximé, figuris \& similitudinibus Liber: non solùm Medicis, sed omnibus rerum Naturae ac Philologiae studiosis, vtilis \& iucundus futurus. Tiguri [Zürich], 7+169 f.

Grande L. 1982. A revision of the fossil genus $†$ Diplomystus, with comments on the interrelationships of clupeomorph fishes. American Museum Novitates 2728: 1-34.

Grande L. 1985. Recent and fossil clupeomorph fishes with materials for revision of the subgroups of clupeoids. Bulletin of the American Museum of Natural History 181(2): 231-372.

Greenwood PH, Rosen DE, Weitzman H, Myers GS. 1966. Phyletic studies of teleostean fishes, with a provisional classification of 
living forms. Bulletin of the American Museum of Natural History 131: 339-455.

Guettard J-E. 1783. Mémoires sur différentes parties des Sciences et Arts, tome 5. Paris : Philippe-Denys Pierres, [iv] $+446+[1] \mathrm{pp}$., $54 \mathrm{pl}$.

Guillaume P-E. 1867. Histoire du diocèse de Toul et de celui de Nancy, depuis l'établissement du christianisme chez les Leuci jusqu'à nos jours, précédée d'une dissertation historique sur l'antiquité de l'église de Toul, tome 4. Nancy: Thomas et Pierron, [iv] $+464 \mathrm{p}$.

Heidtke UHJ. 2007. Die fossilen Zinnoberfische von Münsterappel. Pollichia 23(4): 24-26.

Héron de Villefosse A-M. 1804. Extrait du rapport fait au Conseil des Mines, le 9 pluviôse an XII, sur la partie financière des mines du Hartz, à compter du 12 messidor an XI, jusqu'au 10 nivôse an XII. Journal des Mines 16(95): 395-408.

Huot J-J-N. 1839. Nouveau cours élémentaire de géologie, tome 2. Paris : Librairie encyclopédique de Roret, vi $+794 \mathrm{p}$.

International Commission on Zoological Nomenclature (ICZN). 1999. International Code of Zoological Nomenclature, $4^{\mathrm{e}}$ éd. London: International Trust for Zoological Nomenclature, $306 \mathrm{p}$.

Klipstein PE. 1790. Versuch einer mineralogischen Beschreibung des Vogelsgebirgs in der landgrafschaft Hessen-Darmstadt. Berlin: Friedrich Nicolai, $96 \mathrm{p}$.

Kölbel B, Terken L, Sauerwein M, Sauerwein K, Kölbel S. 2002. Eine fast vergessene Reise Alexander von Humboldts und Steven Jan van Geuns' Reise durch Hessen, die Pfalz, an den Rhein und durch Westfalen im Herbst des Jahres 1789. Cardanus, Jahrbuch für Wissenschaftsgeschichte 3: 79-101.

Labat J-B. 1735. Mémoires du chevalier d'Arvieux, envoyé extraordinaire du Roy à la Porte, Consul d'Alep, d'Alger, de Tripoli \& autres Echelles du Levant, tome 2. Paris : chez CharlesJean-Baptiste Delespine, [iv] $+521+[14] \mathrm{p}$.

Léman S. 1815. Analyse du prétendu plomb phosphaté de Zellerfeld, au Harz par M. Stromeyer; et, à ce sujet, observation sur le plomb sulfaté. Bulletin des Sciences, Société Philomatique de Paris, année 1815: 65-67.

Linnaeus C. 1758. Systema naturae, $10^{\mathrm{e}}$ éd., tome 1. Stockholm: Impensis Laurentii Salvii, $824 \mathrm{p}$.

Lucas J-A-H. 1813. Tableau méthodique des espèces minérales, seconde partie. Paris: D’Hautel, [iv] + xlvii + $587 \mathrm{p}$.

Maraldi. 1705. Des pierres [...] dans lesquelles on trouve des poissons dessechés. Histoire de l'Académie Royale des Sciences avec les Mémoires de Mathématique \& de Physique, année 1703: 22-24.

Mathieu D. 1879. L'ancien régime dans la province de Lorraine et Barrois d'après des documents inédits (1698-1789). Paris : Hachette, [iv] + xii $+469 \mathrm{p}$.

Müller J. 1845. Ueber den Bau und die Grenzen der Ganoiden, und über das natürliche System der Fische. Archiv für Naturgeschichte 11: 91-141.

Münster S. 1550. Cosmographei, oder beschreibung aller länder, herschafften, fürnemsten stetten, geschichten, gebreuche, hantierungen etc. Basel: Petri, [14]+1233 p.

Murray AM, Vernygora O, Japundžić S, Radovčić J, Wilson MVH, Bardack D, Grande T. 2016. Relationships of the species of Armigatus (Clupeomorpha, Ellimmichthyiformes) and the description of a new species from the Cretaceous of Dalmatia, Croatia. Journal of Vertebrate Paleontology 36(6): e1226851.

Murray AM, Wilson MVH. 2013. Two new paraclupeid fishes (Clupeomorpha: Ellimmichthyiformes) from the Late Cretaceous of Morocco. In: Arratia G, Schultze H-P, Wilson MVH, eds. Mesozoic Fishes 5, Global diversity and evolution. Munich: Verlag Dr. Friedrich Pfeil, pp. 267-290.
Nöggerath JJ. 1811. Description minéralogique du gisement de la Braunkohle (houille brune), dans la colline de Putzberg, près de Friesdorf, département de Rhin-et-Moselle, avec diverses observations relatives à toute la formation de cette espèce dans la contrée du Bas-Rhin, traduit de l'allemand par M. Beurard. Journal des Mines 30(179): 335-372.

Nottes G, Heidtke U. 1987. Zur Strahlungsanomalie an fossilen Zinnoberfischen von Münsterappel. Aufschluss 38: 389-393.

Omalius-d'Halloy JBJ d'. 1808. Suite de l'essai sur la géologie du Nord de la France. Journal des Mines 24: 439-466.

Palissy B. 1580. Discours admirables, de la nature des eaux et fonteines, tant naturelles qu'artificielles, des metaux, des sels \& salines, des pierres, des terres, du feu \& des emaux. Paris : Chez Martin le Jeune, [16] +361+[23]p.

Pictet F-J, Humbert A. 1866. Nouvelles recherches sur les poissons fossiles du Liban. Genève: Georg, vii +115 p., 19 pl.

Quérard J-M. 1827. La France littéraire, ou dictionnaire bibliographique des savants, historiens et gens de lettres de la France, ainsi que des littérateurs étrangers qui ont écrit en français, plus particulièrement pendant les $\mathrm{XVIII}^{\mathrm{e}}$ et $\mathrm{XIX}^{\mathrm{e}}$ siècles, tome 1. Paris : Firmin Didot, xxix $+582 \mathrm{p}$.

Sainte-Claire Deville P. 1929. Études historiques sur les mines et les usines des pays de la Sarre sous la Révolution française et le Premier Empire (1792-1815) (suite). Annales des Mines série 12, Mémoires, 15: 5-87.

Sauvage H-E. 1888. Poissons du terrain houiller de Commentry. In: Brongniart C, Sauvage H-E, eds. Études sur le terrain houiller de Commentry, Livre 3, Faunes ichthyologique et entomologique. Saint-Étienne: Société de l'Industrie Minérale, pp. 39-120.

Savardan A. 1836. Traitement des maladies de la peau. Journal des Connaissances Médico-Chirurgicales 3(7): 263-269.

Savardan A. 1846. Monseigneur l'évêque du Mans et le phalanstère ; correspondance avec l'évêché suivie d'un chapitre intitulé «Le Curé », extrait d'un travail inédit ayant pour titre «La Commune rurale; ce qu'elle est et ce qu'elle pourrait être». Paris : Librairie Sociétaire, [ii] $+53 \mathrm{p}$.

Savardan A. 1866. Avenir. Études d'économie sociale. Paris: Librairie des Sciences Sociales, [xiii] $+520 \mathrm{p}$.

Scheuchzer JJ. 1708. Piscium querelae et vindiciae. Tiguri [Zürich]: Gessner, [ii] +36 p., 5 pl.

Smith M. 2015. The Natural History Museum Fossil Fish Collection: Smith Woodward's role in the development and use of this priceless resource. Geological Society, Special Publications 430: 63-85.

Stamberg S. 2013. New data on the osteology of the actinopterygian fish Amblypterus and the relationship between Amblypterus and Paramblypterus. Acta Musei Nationalis Pragae Series B, Historia Naturalis, 69(3-4): 183-194.

Stamberg S. 2018. Actinopterygians of the Permian locality Buxièresles-Mines (Bourbon-l'Archambault Basin, France) and their relationship to other early actinopterygians. Fossil Imprint 74 (3-4): 245-291.

Suckow GA. 1782. Mineralogishe Beobachtungen über einige benachbarte Gegenden. Bemerkungen der Kurpfälzische physikalisch-ökonomische Gesellschaft Jahre 1781: 337-384.

Traquair RH. 1877. On the Agassizian genera Amblypterus, Palaeoniscus, Gyrolepis, and Pygopterus. Quarterly Journal of the Geological Society of London 33: 548-578.

Troschel FH. 1851. Ueber neue fossile Fische von Winterburg. Verhandlungen des Naturhistorischen Vereines der Preussischen Rheinlande und Westphalens 8: 518-542, pl. 9-14.

Tutot J-J. 1779. Académies. Séances de diverses sociétés. L'Esprit des Journaux françois et étrangers $8^{\mathrm{e}}$ année, (5): 276-294. 
Vernygora OV, Murray AM. 2016. A new species of Armigatus (Clupeomorpha, Ellimmichthyiformes) from the Late Cretaceous of Morocco, and its phylogenetic relationships. Journal of Vertebrate Paleontology 36(1): e1031342.

Vernygora OV, Murray AM. 2021. Morphological variation among the species of Armigatus (Teleostei, Clupeomorpha, Ellimmichthyiformes) and new material of Armigatus alticorpus from the Upper Cretaceous (Cenomanian) of Hakel, Lebanon. Cretaceous Research 117: 104601.

Volney C-F. 1787. Voyage en Syrie et en Egypte, pendant les années 1783, 1784 et 1785, tome 1. Paris : Desenne et Volland, xvi +383 p., 2 cartes.

Woodward AS. 1888. Note on some vertebrate fossils from the Province of Bahia, Brazil, collected by Joseph Mawson, Esq., F.G.S. Annals and Magazine of Natural History Series 6, 2: 132-136.
Woodward AS. 1891. Catalogue of the fossil Fishes in the British Museum, Part II. London: British Museum (Natural History), $\mathrm{xli}+567 \mathrm{p} ., 16 \mathrm{pl}$.

Woodward AS. 1901. Catalogue of the fossil Fishes in the British Museum, Part IV. London: British Museum (Natural History), xxxix +636 p., $19 \mathrm{pl}$.

Young MT, Brignon A, Sachs S, Hornung JJ, Foffa D, Kitson JJN, et al. 2020. Cutting the Gordian knot: a historical and taxonomic revision of the Jurassic crocodylomorph Metriorhynchus. Zoological Journal of the Linnean Society 2020: zlaa092.

Woodward J. 1729. A catalogue of the foreign fossils in the collection of J. Woodward M. D. Brought as well from several parts of Asia, Africa, and America; as from Sweden, Germany, Hungary, and other parts of Europe, part 2. London: F. Fayram, iv $+33 \mathrm{p}$.

Citation de l'article : Brignon A. 2021. Les «ichtyolites» (Actinopterygii) de la collection Jean-Baptiste Beurard (1745-1835) : intérêt historique et redécouverte de la série type d'Armigatus brevissimus (Blainville, 1818) du Cénomanien du Liban, BSGF - Earth Sciences Bulletin 192: 34. 